\title{
Metabolic analyses elucidate non-trivial gene targets for amplifying dihydroartemisinic acid production in yeast
}

\section{Ashish Misra ${ }^{1 \dagger}$, Matthew F. Conway ${ }^{1 \dagger}$, Joseph Johnnie ${ }^{2}$, Tabish M. Qureshi ${ }^{1}$, Bao Lige $^{3}$, Anne M. Derrick $^{3}$, Eddy C. Agbo $^{3}$ and Ganesh Sriram ${ }^{1}$ *}

\author{
Department of Chemical and Biomolecular Engineering, University of Maryland, College Park, MD, USA \\ ${ }^{2}$ Institute for Systems Engineering, University of Maryland, College Park, MD, USA \\ ${ }^{3}$ Fyodor Biotechnologies, Baltimore, MD, USA
}

\section{Edited by: \\ David Nielsen, Arizona State \\ University, USA}

\section{Reviewed by:}

Stefan Junne, Technische Universität Berlin, Germany

Yinjie Tang, Washington University, USA

\section{*Correspondence:}

Ganesh Sriram, Department of Chemical and Biomolecular Engineering, University of Maryland, 1208D, Chemical and Nuclear Engineering Building 090, College Park, MD 20742, USA

e-mail: gsriram@umd.edu

\section{${ }^{\dagger}$ Present address:}

Ashish Misra, DBT-ICT Centre for Energy Biosciences, Institute of Chemical Technology, Mumbai, India; Matthew F. Conway, Department of Chemical Engineering, Columbia University, New York, NY, USA.
Synthetic biology enables metabolic engineering of industrial microbes to synthesize valueadded molecules. In this, a major challenge is the efficient redirection of carbon to the desired metabolic pathways. Pinpointing strategies toward this goal requires an in-depth investigation of the metabolic landscape of the organism, particularly primary metabolism, to identify precursor and cofactor availability for the target compound. The potent antimalarial therapeutic artemisinin and its precursors are promising candidate molecules for production in microbial hosts. Recent advances have demonstrated the production of artemisinin precursors in engineered yeast strains as an alternative to extraction from plants. We report the application of in silico and in vivo metabolic pathway analyses to identify metabolic engineering targets to improve the yield of the direct artemisinin precursor dihydroartemisinic acid (DHA) in yeast. First, in silico extreme pathway (ExPa) analysis identified NADPH-malic enzyme and the oxidative pentose phosphate pathway (PPP) as mechanisms to meet NADPH demand for DHA synthesis. Next, we compared key DHA-synthesizing ExPas to the metabolic flux distributions obtained from in vivo ${ }^{13} \mathrm{C}$ metabolic flux analysis of a DHA-synthesizing strain. This comparison revealed that knocking out ethanol synthesis and overexpressing glucose-6-phosphate dehydrogenase in the oxidative PPP (gene YNL241C) or the NADPH-malic enzyme ME2 (YKLO29C) are vital steps toward overproducing DHA. Finally, we employed in silico flux balance analysis and minimization of metabolic adjustment on a yeast genome-scale model to identify gene knockouts for improving DHA yields. The best strategy involved knockout of an oxaloacetate transporter (YKL120M) and an aspartate aminotransferase (YKL106W), and was predicted to improve DHA yields by 70 -fold. Collectively, our work elucidates multiple non-trivial metabolic engineering strategies for improving DHA yield in yeast.

Keywords: artemisinin, metabolic engineering, metabolic pathway, extreme pathway, isotope labeling, metabolic flux analysis, flux balance analysis, minimization of metabolic adjustment

\section{INTRODUCTION}

Artemisinin-based combination therapy (ACT) is currently the most commonly used treatment for malaria (Weathers et al., 2006; Eastman and Fidock, 2009; Price and Douglas, 2009), an infectious disease that is widespread particularly in regions of Africa, Asia, and South America (Feachem et al., 2010; O’Meara et al., 2010). Artemisinin, the primary component of ACT, is a naturally produced sesquiterpene lactone endoperoxide. Artemisinin and its derivatives have also been found to exhibit anti-cancer properties (Ferreira etal., 2010) such as inducing apoptosis in lung cancer cells (Gao et al., 2013) and preventing cell proliferation in breast cancer cells (Ba et al., 2012; Zhang et al., 2013). In nature, artemisinin is synthesized by the plant Artemisia annua through conversion of the intermediate sesquiterpene farnesyl pyrophosphate (FPP; Bertea et al., 2005; Liu et al., 2011). The precursor FPP is synthesized from the primary metabolite acetyl-coenzyme A (COA) via the mevalonate (MVA) pathway, or from glyceraldehyde-3-phosphate (GAP) and pyruvate via the methylerythritol phosphate (MEP) pathway (Figure 1). A. annua has been the primary source for meeting almost all the worldwide demand for artemisinin, despite producing artemisinin up to less than $1.0-1.5 \%$ of its dry weight (Kindermans et al., 2007; Covello, 2008). Not surprisingly, the price of artemisinin has varied substantially from a lower bound of US\$150-170 $\mathrm{kg}^{-1}$ to an upper bound of US\$1,100-1,500 kg-1 (World Health Organization, 2010), partially due to variability in the cultivation of $A$. annua. Therefore, it is necessary to explore avenues for reliable production of artemisinin that offer this drug at the minimal possible cost to developing countries (Covello, 2008). To achieve this goal, researchers have demonstrated the synthesis of artemisinin precursors in microbes (Ro et al., 2006; Zhang et al., 2008; Westfall et al., 2012; Paddon et al., 2013) or plants (e.g., Zhang et al., 2011) engineered to express genes from the A. annua artemisinin pathway, thereby enabling conversion of endogenously produced FPP to artemisinin precursors. Orthogonally, chemical syntheses for artemisinin from starting materials ranging from natural 


\section{A}

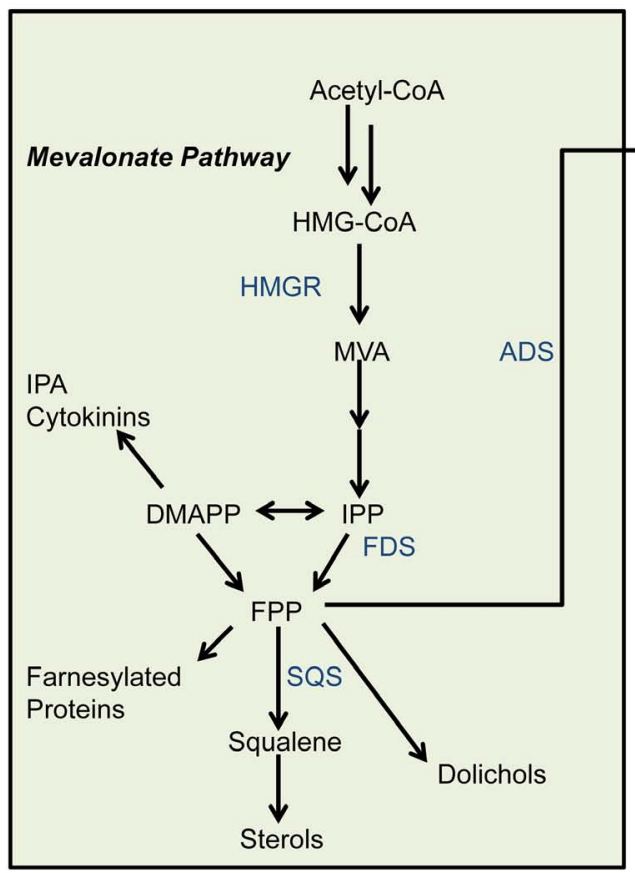

B

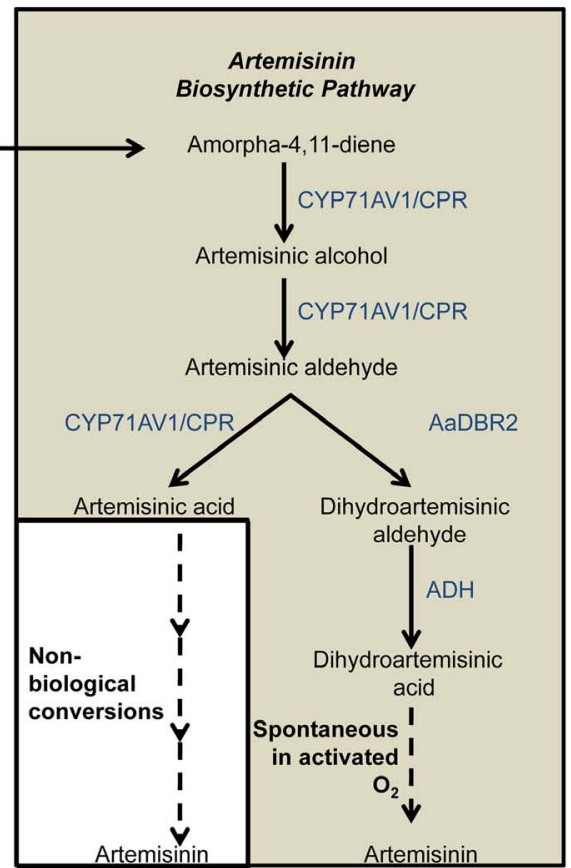

FIGURE 1 | Artemisinin precursor synthesis pathways in yeast. In the native isoprenoid biosynthesis pathway in yeast (A), IPP synthesized via the MVA pathway is converted to FPP. HMGR is a key enzyme in the isoprenoid biosynthetic pathway that feeds the artemisinin precursor synthesis pathway (B). Steps that are not known to be catalyzed by enzymes are depicted with dashed lines. Enzyme and metabolite name abbreviations: AaDBR2, A. annua double bond reductase; $A D H$, (dihydroartemisinic) aldehyde dehydrogenase;
ADS, amorpha-4,11-diene synthase; CYP71AV1, cytochrome P450 monooxygenase; CPR, A. annua cytochrome $\mathrm{P} 450$ reductase; CoA, coenzyme A; DMAPP, dimethylallyl diphosphate; FDS, farnesyl diphosphate synthase; FPP, farnesyl pyrophosphate; HMG-CoA, 3-hydroxy-3-methylglutaryl-CoA; HMGR, HMG-CoA reductase; IPA, isopentenyladenine; IPP, isopentenyl diphosphate; MVA, mevalonate; SOS, squalene synthase. Adapted from Zhang et al. (2008) and Teoh et al. (2006). terpenoids (reviewed in Covello, 2008) to cyclohexenone (e.g., Zhu and Cook, 2012) have been reported.

The first applications of synthetic biology to artemisinin production were those of Keasling and colleagues (Ro et al., 2006) and of Covello and colleagues (Teoh et al., 2006). Both groups reported the engineering of Saccharomyces cerevisiae to produce artemisinic acid (AA), which can be converted to artemisinin through a sequence of chemical steps. The breakthrough work by Keasling and colleagues was followed by several reports of significantly improved AA titers from the same group (Westfall et al., 2012; Paddon et al., 2013; see detailed description below). Independently, Covello and colleagues demonstrated the production of dihydroartemisinic acid (DHA) in S. cerevisiae (Zhang et al., 2008). As an artemisinin precursor, DHA is preferable to AA for multiple reasons. Firstly, DHA can be oxidized to artemisinin spontaneously without the involvement of enzymes (Sy and Brown, 2002; Brown and Sy, 2004). In planta artemisinin biosynthesis is hypothesized to proceed through DHA via this mechanism (Bertea et al., 2005), circumstantial evidence for which comes from the observation that DHA-rich chemotypes of $A$. annua exhibit significantly higher artemisinin production than AA-rich chemotypes (Wallaart etal., 2000; Rydén et al., 2010). Secondly, semi-synthetic routes to artemisinin production have relied upon DHA as the starting material (e.g., Lévesque and Seeberger, 2012; Westfall et al., 2012). Thus, the possibility of engineering DHA biosynthesis in microbes opens up an alternative route for artemisinin synthesis in yeast, which has the potential to be executed completely in vivo (Zhang et al., 2008).

The AA route for artemisinin production has recently seen impressive scientific success and commercialization (Hale et al., 2007; Chandran et al., 2008; Lenihan et al., 2008; Westfall et al., 2012; Paddon et al., 2013). The titers and yields of artemisinin or its precursors have been improved substantially by optimization of downstream metabolic pathways as well as process development. The work of Keasling and coworkers improved FPP production, and thereby AA titers, in the initial AA-synthesizing strains (Ro et al., 2006) by ingeniously combining several approaches. These included (i) overexpression of $u p c 2-1$, a transcription factor involved in the regulation of sterol production, to increase flux through the MVA pathway; (ii) downregulation of squalene synthase (ERG9), which diverts FPP away from DHA production to sterol production; and (iii) overexpression of 3-hydroxy3-methylglutaryl (HMG)-CoA reductase (HMGR) to enhance conversion of HMG-CoA to MVA. By employing these strategies in concert, Keasling and colleagues were able to improve the titer of amorpha-4,11-diene, a precursor of the artemisinin pathway (Figure 1), by more than 15-fold over the original AA-synthesizing strain (Ro et al., 2006). Further work (Lenihan et al., 2008) improved AA titers of these strains through process optimization of fermentation parameters. Recently, Keasling and 
colleagues employed the strategy of overexpressing all enzymes of the MVA pathway together with repression of ERG9 to achieve very high titers and yields of amorpha-4,11-diene and AA (Westfall et al., 2012). While all previous attempts employed galactose or glucose as the sole carbon source, Westfall etal. (2012) fed ethanol as a second carbon source and obtained yields of amorpha-4,11-diene higher than $18 \mathrm{C} \mathrm{mol} \mathrm{\%} \mathrm{(Figure} \mathrm{2).} \mathrm{More}$ recently, Paddon etal. (2013) improved AA titers by more than 15 -fold to $25 \mathrm{~g} \mathrm{~L}^{-1}$. Coupled with their development of a scalable chemical process for conversion of AA to artemisinin, this work is a major breakthrough in the commercial production of artemisinin.

The approaches described above have improved AA yields almost exclusively by manipulating flux through downstream pathways, specifically the MVA and sterol biosynthesis pathways. However, another powerful approach to enhance the production of artemisinin precursors would be to engineer upstream (primary and central carbon) metabolism. Several computational and experimental tools such as extreme pathway (ExPa) analysis (Wiback and Palsson, 2002; Llaneras and Picó, 2010), flux balance analysis (FBA; Orth et al., 2010), minimization of metabolic

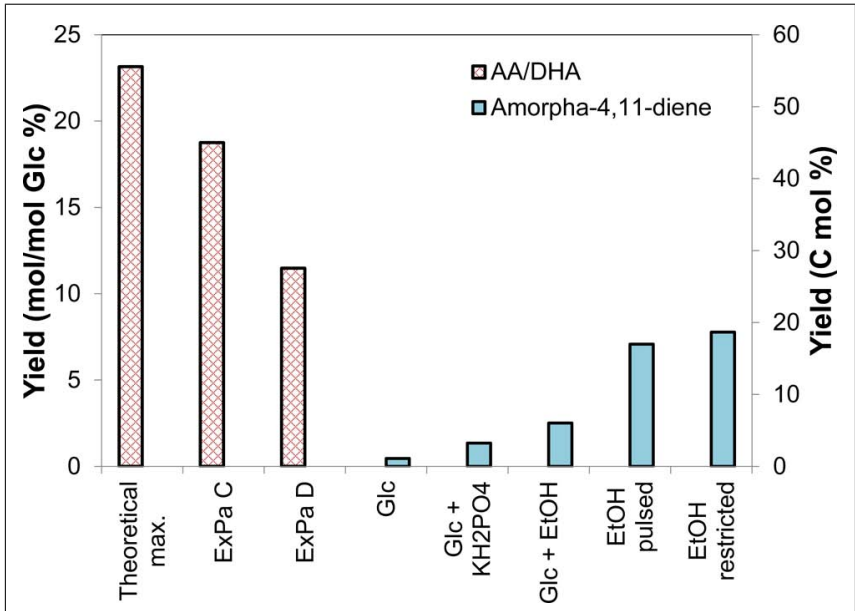

FIGURE 2 | Calculated (red bars) and previously reported (blue bars) yields of artemisinin precursors. The theoretical maximum yield of the artemisinin precursors amorpha-4,11-diene, AA, or DHA is $22 \mathrm{~mol} / 100 \mathrm{mo}$ glucose (Glc) (left axis) or $55 \mathrm{C} \mathrm{mol} \mathrm{\%} \mathrm{(right} \mathrm{axis).} \mathrm{One} \mathrm{ExPa} \mathrm{(ExPa} \mathrm{C,} \mathrm{with}$ ME2 active), yields $18 \mathrm{~mol} D H A / 100$ mol glucose), with concomitant biomass production (3 mol/100 mol glucose). Here, ME2 supplies reductant to meet the NADPH requirement. Another ExPa (ExPa D, with an active oxidative PPP), yields $11 \mathrm{~mol} \mathrm{DHA} / 100 \mathrm{~mol}$ glucose, with concomitant biomass production ( $6 \mathrm{~mol} / 100 \mathrm{~mol}$ glucose). Here, the oxidative PPP supplies reductant to meet the NADPH requirement. Figure 3 depicts flux distributions through these ExPas. The yields of artemisinin precursors in the pioneering microbial production studies (Ro etal., 2006; Zhang etal., 2011) were relatively low; however, subsequent work obtained further improvement in amorpha-4,11-diene and AA yield by increasing flux through the MVA pathway and varying feed composition. Recently, Westfall et al. (2012) achieved drastic improvement in amorpha-4,11-diene yield of close to $20 \mathrm{C} \mathrm{mol} \%$ by using an optimized yeast strain with a mixed feed consisting of ethanol (EtOH) and Glc. They achieved yields of $1.1 \mathrm{C} \mathrm{mol} \%$ with glucose as carbon source, which increased to $3.2 \mathrm{C} \mathrm{mol} \%$ by using $\mathrm{KH}_{2} \mathrm{PO}_{4}$ with the glucose feed, to $6.0 \mathrm{C} \mathrm{mol} \%$ by using glucose and $\mathrm{EtOH}$ mixed feed. The highest yields obtained were 17.0 and $18.7 \mathrm{C} \mathrm{mol} \mathrm{\%} \mathrm{with} \mathrm{a}$ restricted $\mathrm{EtOH}$ feed. adjustment (MOMA) analysis (Segrè et al., 2002) as well as ${ }^{13} \mathrm{C}$ metabolic flux analysis ( ${ }^{13} \mathrm{C}$ MFA; e.g., Feng et al., 2010; Papini etal., 2012; Ahn and Antoniewicz, 2013; Nargund and Sriram, 2013) are now available to dissect metabolism, derive insight and propose metabolic engineering strategies. Because primary metabolic pathways supply substantial amounts of carbon and reductant to downstream pathways such as MVA and sesquiterpene synthesis, such strategies may identify non-trivial metabolic engineering strategies and thus play a very significant role in improving flux to AA or DHA. There have been relatively few reports of computationally inferred genetic engineering strategies in upstream metabolism for improving microbial yields of intermediary or secondary metabolites. Studies that have successfully accomplished this include those focusing on the production of L-valine (Park et al., 2007), lycopene (Alper et al., 2005), and $\mathrm{C}_{14}-\mathrm{C}_{16}$ fatty acids (Ranganathan et al., 2012) by Escherichia coli. In one study directed at secondary metabolite production by yeast, Asadollahi et al. (2009) identified genetic interventions toward increased production of sesquiterpenes synthesized via the MVA pathway, by in silico analyses of a genome-scale model of yeast metabolism. They predicted that knocking out glutamate dehydrogenase $(G D H 1)$, whose product assimilates nitrogen at the expense of $\mathrm{NADPH}$, will result in a 10 -fold increase in the production of the sesquiterpene cubebol. Mechanistically, this knockout was predicted to shunt carbon via an alternative reaction that consumes NADH instead of NADPH, thus improving NADPH availability to the MVA pathway and sesquiterpene synthesis. Indeed, on in vivo implementation, the GDH1 knockout strategy led to a significant ( $\sim$ twofold) increase in the titer of cubebol.

Because modeling approaches are immensely useful in synthetic biology (Zheng and Sriram, 2010), this article investigates strategies for improving DHA yields in engineered yeast by employing a variety of computation-assisted methodologies. These include ExPa analysis, ${ }^{13} \mathrm{C}$ MFA, FBA, and MOMA analysis. ExPa and elementary flux mode analysis enable detailed investigation of the flux distribution space (phenotypic space) of a metabolic network (Schilling et al., 2000) to obtain insights on the network's capabilities and limitations. Such analyses can suggest genetic intervention strategies to effect a desired outcome from the network Becker et al. (2011). ${ }^{13} \mathrm{C}$ MFA is a powerful methodology currently used for estimating intracellular fluxes. In this methodology, isotope labeling signatures obtained from feeding a mixture of ${ }^{13} \mathrm{C}$ and ${ }^{12} \mathrm{C}$ carbon sources to an organism, in conjunction with extracellular flux measurements, are used to evaluate intracellular flux distributions (e.g., Wiechert, 2001; Sriram et al., 2004, 2008; Murphy et al., 2013). FBA, a complementary approach to ${ }^{13} \mathrm{C}$ MFA, optimizes a metabolic objective to estimate or predict flux distribution seven at the genome-scale (Grafahrend-Belau et al., 2009; Orth et al., 2010). In genome-scale FBA, an organism's inventory of metabolic reactions is used to assemble a stoichiometric matrix. Thermodynamic irreversibility constraints as well as measurements of a few extracellular fluxes such as substrate uptake and product secretion are used to constrain the null space of this matrix, thus generating a phenotypic space of feasible flux distributions. An objective function such as maximization of biomass production is then employed to isolate a particular solution within 
the feasible solution space. Several extensions of the FBA approach enable prediction of changes in flux distributions due to genetic interventions, including OptKnock (Burgard et al., 2003), OptStrain (Pharkya et al., 2004), OptForce (Ranganathan et al., 2010), and MOMA (Segrè et al., 2002). MOMA predicts flux alterations resulting from gene knockouts, and thereby enables identification of advantageous knockouts. It is based on the premise that gene knockouts restrict the wild type phenotypic space of an organism, and that organisms respond by minimally adjusting their wild type flux distribution to make it lie within the restricted phenotypic space (Segrè et al., 2002). This contrasts with FBA, which assumes that organisms respond by finding a new optimum of their objective function within the restricted phenotypic space.

To our knowledge, this is the first study utilizing a variety of computational and experimental pathway analysis techniques to identify metabolic engineering targets for enhanced production of artemisinin precursors in yeast. Together, our analyses elucidate upstream bottlenecks in DHA synthesis and suggest non-trivial genetic intervention strategies for pushing carbon toward the DHA pathway and thereby improving DHA yield.

\section{MATERIALS AND METHODS ExPa ANALYSIS}

For ExPa analysis, we constructed a metabolic model of yeast engineered to synthesize DHA. This model consisted of central carbon metabolic pathways native to yeast including glycolysis, pentose phosphate pathway (PPP), tricarboxylic acid (TCA) cycle, MVA pathway, pathways for synthesis of biomass components and a consolidated reaction encompassing the pathway from MVA to DHA. Altogether, this model contained 63 reactions and 51 metabolites. To determine ExPas, we analyzed this model with the expa program from Bell and Palsson (2004). The resulting ExPas are tabulated in Data Sheet 1 in Supplementary Material.

\section{CELL CULTURE, STRAIN CHARACTERIZATION, AND METABOLIC MEASUREMENTS}

\section{Yeast cell culture in microplates}

We used three $S$. cerevisiae strains in this study (FyAA, FyDHA, and $\mathrm{Fy}_{0}$; Zhang et al., 2008), all of which were designer yeast strains carrying lysine and methionine auxotrophies. Strain FyAA was engineered with genes for the synthesis of AA, specifically $A$. annua farnesyl diphosphate synthase (FDS), amorpha-4,11-diene synthase (ADS), cytochrome P450 monooxygenase (CYP71AV1), and $A$. annua cytochrome P450 reductase $(C P R)$. Strain FyDHA was engineered with genes for the synthesis of DHA, specifically FDS, ADS, CYP71AV1, CPR, and A. annua double bond reductase $(A a D B R 2)$. Figure 1 depicts the reactions catalyzed by the products of these genes. Strain Fy $\mathrm{F}_{0}$ was an empty vector control. Further genetic engineering details are provided in the supplementary material of Zhang et al. (2008). We grew the strains in synthetic defined base yeast minimal medium (Clontech Laboratories, Mountain View, CA, USA) supplemented with trace amounts of lysine and methionine due to the auxotrophies in the strains. This medium was supplemented with $2 \%$ $(\mathrm{w} / \mathrm{v})$ glucose or galactose as carbon source. All growth and ${ }^{13} \mathrm{C}$ MFA experiments were performed on $2 \mathrm{~mL}$ batch cultures in deep-well microplates in a Biotek Synergy HT microplate reader (Biotek Instruments, Winooski, VT, USA) at $30^{\circ} \mathrm{C}$ with continuous shaking. To induce DHA or AA production, cultures grew for $45-55 \mathrm{~h}$ on this medium supplemented with $2 \%(\mathrm{w} / \mathrm{v})$ galactose as the carbon source. For isotope labeling studies, we used either $20 \% \mathrm{U}_{-}{ }^{13} \mathrm{C}$ galactose or $100 \% 1-{ }^{13} \mathrm{C}$ galactose in parallel labeling experiments. To harvest cells we centrifuged the cell suspensions, separated the supernatant and immediately froze the pellets in liquid nitrogen to arrest metabolism. The pellets and supernatants were lyophilized and stored at $-80^{\circ} \mathrm{C}$ until further analysis.

\section{Strain characterization}

Expression of recombinant genes in the engineered strains was confirmed by RT-PCR, using primers from Zhang et al. (2008). A. annua actin 1 was used as a negative control, whereas the yeast ACT1 and TAF10 were used as positive controls (Teste et al., 2009). Primers (sequences are listed in Data Sheet 2 in Supplementary Material) were obtained from Integrated DNA Technologies (Coralville, IA, USA).

Cell growth rates were determined via online optical density measurements on the Biotek microplate reader. Measurements for glucose, ethanol, and glycerol were performed by analyzing the supernatant with a YSI 2700 Select metabolite analyzer (YSI Life Sciences, Yellow Springs, OH, USA), using a measurement kit appropriate for each metabolite. These measurements are listed in Data Sheet 2 in Supplementary Material. AA and DHA were extracted by using protocols adapted from Zhang et al. (2008) and quantified by gas-chromatography-mass spectrometry (GCMS) of their TMS derivatives, using methyl stearate as an internal standard.

\section{Extraction and analysis of proteinogenic amino acids for ${ }^{13} \mathrm{C}$ isotopomer measurements}

Proteinogenic amino acids in cell pellets were obtained by vacuumhydrolyzing the pellets with $6 \mathrm{~N}$ hydrochloric acid (Thermo Scientific, Rockford, IL, USA). The hydrolysate was mixed with a known amount of norleucine as an internal standard and derivatized by adding $100 \mu \mathrm{L} N$-(tert-butyldimethylsilyl)$N$-methyltrifluoroacetamide (MTBSTFA; Thermo Scientific) in $100 \mu \mathrm{L}$ dimethylformamide (Thermo Scientific) and heating at $70^{\circ} \mathrm{C}$ for $1 \mathrm{~h}$. GC-MS analysis of $1 \mu \mathrm{L}$ of this derivatized mixture was performed using previously published instrument methods (Sriram et al., 2008) on a Varian 300MS quadrupole GC-MS unit (Bruker, Billerica, MA, USA) equipped with a VF5-ms column of dimensions $0.25 \mathrm{~mm} \times 30 \mathrm{~m} \times 0.25 \mu \mathrm{m}$ and capable of detecting ions by electron ionization. Quantification of amino acids in the biomass was performed by comparing the chromatographic peak of each amino acid with that of the norleucine internal standard. Isotopomer abundances obtained from the mass spectrum of each amino acid were processed to filter out natural abundances of elements other than metabolic carbon, using a previously developed in-house MATLAB program, whose accuracy has been verified by using amino acid isotopomer mixtures of known isotopomeric composition (Sriram et al., 2008). Isotopomer abundances for the three strains are reported in Data Sheet 2 in Supplementary Material. 


\section{Reproducibility and statistics}

All experiments were performed with two or three biological replicates, and statistical significance was determined by calculating $p$-values through a Student's $t$-test.

\section{Evaluation of metabolic fluxes from ${ }^{13} \mathrm{C}$ isotopomer data}

We evaluated fluxes from the ${ }^{13} \mathrm{C}$ mass isotopomer data and extracellular flux measurements by using our flux evaluation program NMR2Flux + (Sriram et al., 2004, 2008) to fit this data to a steady state flux-isotopomer model. This model consisted of yeast central carbon metabolic pathways, including glycolysis, PPP, TCA cycle, and anaplerotic pathways. For the retrobiosynthetic reconstruction of metabolic precursor mass isotopomer distributions (MIDs) from the amino acid MIDs (e.g., pyruvate from alanine or oxaloacetate from aspartic acid), we used standard pathways from the precursors to the amino acids (Szyperski, 1995, 1998). These pathways are universally conserved across life (Romano and Conway, 1996). We used standard models for the biosynthesis of amino acids from the intermediates of these pathways (e.g., Szyperski, 1995). We found that labeling of the proteinogenic amino acids except methionine and lysine was very close to $20 \%$ for cells grown on $20 \%$ U- ${ }^{13} \mathrm{C}$ galactose, which indicates attainment of isotopic steady state (data not shown). However, the metabolism is still at pseudo-steady state due to the batch culture. Statistical analysis was performed by the NMR2Flux + program, using a bootstrap Monte Carlo simulation to execute 100 runs of the flux evaluation by perturbing measurements by their known standard error, as previously described (Sriram et al., 2004, 2008). Evaluated fluxes for the three strains are reported in Data Sheet 2 in Supplementary Material.

\section{FBA AND MOMA ANALYSIS}

We modified the iMM904 genome-scale model for S. cerevisiae (Mo et al., 2009) by appending it with the reactions necessary to synthesize DHA from FPP. We constrained this model with the measured extracellular uptake fluxes (glucose or galactose) as well as intracellular fluxes estimated from the ${ }^{13} \mathrm{C}$ isotopomer data. The open-source COBRA toolbox (Schellenberger et al., 2011) was used to perform FBA and MOMA analysis on the model. For FBA, we used maximization of biomass growth as the objective function. We performed MOMA to assess the effects of all possible single-gene knockouts on DHA production. For this, we used an in-house MATLAB script (available on request) to automate certain repetitive steps such as recurring calls to COBRA. The non-lethal single-gene knockouts that significantly increased DHA yields were used in a second iteration of the MOMA analysis to identify double-gene knockouts that enhanced DHA production. The results of this analysis are available in Data Sheet 3 in Supplementary Material.

\section{RESULTS AND DISCUSSION}

The currently available synthesis routes for DHA (or AA) use galactose or glucose as carbon sources (Ro et al., 2006; Teoh et al., 2006; Zhang et al., 2008; Paddon et al., 2013). Strategizing how to produce DHA from these substrates at high yield is a complex problem, solving which requires answering the following questions. (i) What is the theoretical maximal yield of DHA on glucose or galactose? (ii) Is this yield limited by the availability of reductant cofactors? (iii) Which configuration of pathways and fluxes favors a high yield? (iv) Do current DHA-synthesizing strains operate near or distantly from such a configuration? (v) Which minimal sets of genetic interventions can drastically improve the yield of a low DHA-producing strain? Below, we describe our computational analyses (ExPa analysis and MOMA analysis) and experimental investigations $\left({ }^{13} \mathrm{C}\right.$ MFA) toward answering these questions.

\section{THEORETICAL MAXIMAL YIELD OF DHA OR AA ON GLUCOSE}

The value of the maximal yield of DHA on glucose (or a similar sugar such as galactose) depends on whether the reductant cofactors NADH and NADPH are considered in the analysis, and whether a distinction is made between the cofactors NADH and NADPH. A cofactor-free theoretical yield calculation reveals that yeast can produce a maximum yield of $22 \mathrm{~mol}$ DHA/100 mol glucose, or 1 mol DHA per $4.5 \mathrm{~mol}$ glucose (Figure 2). Metabolically, this would proceed as follows. Glycolysis would convert $4.5 \mathrm{~mol}$ glucose to $9 \mathrm{~mol}$ acetyl-CoA, which the MVA pathway would condense to form $3 \mathrm{~mol}$ dimethylallyl diphosphate (DMAPP). DMAPP would further condense to form FPP in a molar proportion of 3 DMAPP:1 FPP. FPP would then be converted to amorphadiene and ultimately to DHA in a $1: 1$ molar proportion (Bertea et al., 2005). This DHA yield is equivalent to $55 \mathrm{C} \mathrm{mol} \mathrm{\% ,}$ wherein the $45 \mathrm{C} \mathrm{mol} \mathrm{\%} \mathrm{carbon} \mathrm{loss} \mathrm{is} \mathrm{due} \mathrm{to} \mathrm{the} \mathrm{release} \mathrm{of} \mathrm{CO}_{2}$ in multiple pathway steps. This calculation and yield value also apply to AA production from glucose or galactose.

The fact that artemisinin, DHA and AA are significantly reduced compounds necessitates the consideration of reductant (cofactor) demand and supply in this yield calculation. In the upstream section of the pathway described above, the conversion of $4.5 \mathrm{~mol}$ glucose to $9 \mathrm{~mol}$ acetyl-CoA supplies $18 \mathrm{~mol} \mathrm{NADH}$. In the downstream section, 6 mol NADPH is required for the conversion of $9 \mathrm{~mol}$ acetyl-CoA to 3 mol DMAPP. Therefore, if no distinction is made between the NADH and NADPH, this pathway is self-sufficient in reductant because the demand of $6 \mathrm{~mol}$ $\mathrm{NADPH}$ is met by a supply of $18 \mathrm{~mol} \mathrm{NADH}$.

The interchangeability of NADH and NADPH invoked in the above analysis is contingent upon the availability of a transhydrogenase enzyme that converts NADH to NADPH (Stephanopoulos et al., 1998). However, there is no evidence for a transhydrogenase in yeast (Bruinenberg et al., 1983; Nissen et al., 2001). Therefore, other NADPH-supplying pathways will have to be recruited to make DHA production feasible. This complicates the theoretical yield calculation for three reasons. Firstly, multiple pathways including the oxidative PPP and anaplerotic cycles can supply NADPH either by themselves or in combination. Secondly some of these pathways, such as the oxidative PPP, supply NADPH at the expense of carbon, so that recruiting them for NADPH provision can compromise the maximal yield of DHA. Thirdly, the practical necessity of concurrently producing biomass with DHA will obviously reduce the maximal yield of DHA. To analyze this complex set of possibilities and delineate all possible pathway configurations for DHA synthesis with or without cofactor requirement, we performed ExPa analysis of the underlying metabolic network. 


\section{EXPa ANALYSIS OF DHA SYNTHESIS NETWORK}

Colloquially, an ExPa represents one way of "walking through a metabolic network." Mathematically, ExPas are the edges of the polyhedral cones that represent the hyperdimensional phenotypic space that a metabolic network can occupy (Bell and Palsson, 2004; Palsson, 2006). ExPa analysis has previously been applied to a variety of systems on different scales from small scale networks of red blood cells to genome-scale pathway analysis of the influenza virus to elucidate important metabolic properties of the systems (Schilling and Palsson, 2000; Wiback and Palsson, 2002). Amongst the three types of ExPas (types I, II, and III) classified by Palsson (2006), we are interested in types I and III. Type I ExPas effect the conversion of a substrate or substrates to a product or products along with the transport of the substrates and products across the cell membrane. Type III ExPas involve intracellular cycles with no transport across the cell membrane. To perform ExPa analysis, we constructed a simplified (less than genome-scale) metabolic model of yeast capable of producing both DHA and biomass (Data Sheet 1 in Supplementary Material). This model includes 63 reactions including exchange reactions and 51 metabolites including cofactors. We then performed ExPa analysis on the model by (i) neglecting and (ii) considering cofactor requirements to gain insights about the capabilities of the network. Table 1 presents a summary of ExPa families for both cases, and Figure 3 depicts flux distributions through four selected ExPas.
Of these, ExPa A was obtained from a cofactor-free model, and ExPas B, C, and D were obtained by considering cofactor requirements.

\section{COFACTOR-FREE EXPa ANALYSIS REVEALS IMPORTANT CLASSES OF PATHWAYS TOWARD DHA SYNTHESIS}

A cofactor-free ExPa analysis of a network, although an incomplete depiction of the network, permits the delineation of major classes of metabolic routes through it. Such an analysis of the metabolic model in Data Sheet 1 in Supplementary Material revealed 50 type I, 6 type II, and 18 type III ExPas (Table 1 and Data Sheet 1 in Supplementary Material). Of these, only five ExPas synthesized DHA as their sole product, whereas the rest synthesized various secreted metabolites (e.g., glycerol, ethanol, and acetate), biomass or a combination of biomass and secreted metabolites. Interestingly, none of the biomass-producing ExPas concurrently synthesized DHA (Table 1). The DHA yield in the DHA-synthesizing ExPa A was equal to the previously calculated theoretical maximum of $22 \mathrm{~mol}$ DHA/100 mol glucose (Figure 3A).

\section{INCLUSION OF COFACTOR REOUIREMENT RECRUITS NADPH-MALIC ENZYME (ME2) OR THE OXIDATIVE PPP FOR NADPH PROVISION TO DHA SYNTHESIS PATHWAY}

We repeated the foregoing ExPa analysis for a more realistic scenario that considered cofactor requirements and did not include a

Table 1 | Summary of ExPa families for a yeast strain synthesizing DHA from glucose, in the absence and presence of cofactor requirement.

\begin{tabular}{|c|c|c|c|c|c|c|}
\hline \multirow{2}{*}{$\frac{\text { ExPa family }^{\mathbf{a}}}{\text { DHA production }^{b}}$} & \multicolumn{3}{|c|}{ Cofactor-free } & \multicolumn{3}{|c|}{ With cofactor requirement } \\
\hline & $\begin{array}{l}\text { \# of ExPas } \\
5\end{array}$ & \multicolumn{2}{|c|}{ Maximal yields } & $\begin{array}{l}\text { \# of ExPas } \\
238\end{array}$ & \multicolumn{2}{|c|}{ Maximal yields } \\
\hline & (\#1 to \#5) & & & (\#1 to \#238) & & \\
\hline DHA and biomass production ${ }^{\mathrm{C}}$ & 0 & $\mathrm{~N} / \mathrm{A}$ & & 94 & DHA: & 18 \\
\hline & (\#6 to \#16) & & & (\#333 to \#715) & & \\
\hline \multirow[t]{2}{*}{ All carbon lost to $\mathrm{CO}_{2}$} & 10 & $\mathrm{CO}_{2}:$ & 600 & 108 & $\mathrm{CO}_{2}:$ & 600 \\
\hline & (\#17 to \#26) & & & (\#716 to \#823) & & \\
\hline Metabolite production via glycolysis & 10 & Glycerol: & 200 & 83 & Glycerol: & 164 \\
\hline \multirow[t]{2}{*}{ Type II ExPas } & 6 & $\mathrm{~N} / \mathrm{A}$ & & 0 & N/A & \\
\hline & (\#51 to \#56) & & & & & \\
\hline \multirow[t]{2}{*}{ Type III ExPas } & 18 & $\mathrm{~N} / \mathrm{A}$ & & 19 & N/A & \\
\hline & (\#57 to \#74) & & & (\#1068 to \#1086) & & \\
\hline
\end{tabular}

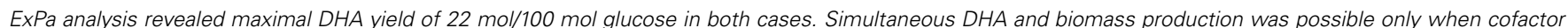

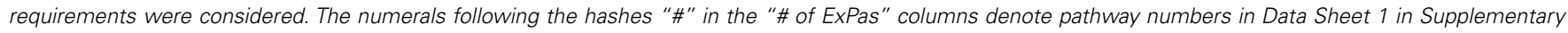
Materials. All yields are in mol/100 mol glucose.

a ExPas are type I unless otherwise specified. See Palsson (2006) and text for definitions of ExPa types.

bNADPH corresponding to maximal DHA yield is supplied by the ME2 reaction; supply of NADPH by the PPP results in lower yields.

${ }^{C}$ This is the most important ExPa family, as it provides insights into strategies for improving overall DHA yield. 


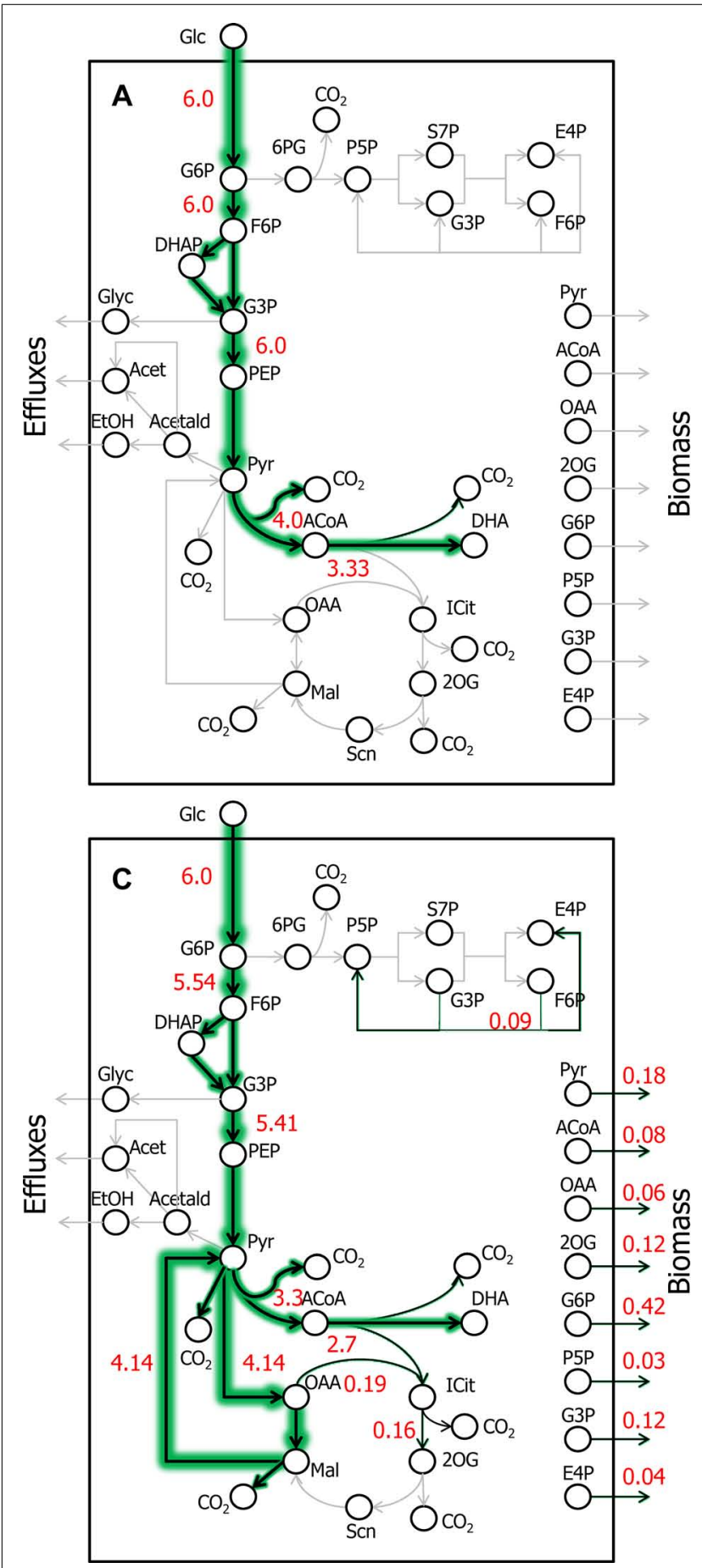

FIGURE 3 | Key DHA- or AA-synthesizing ExPas in yeast. Four ExPas that synthesize DHA (or AA) and/or biomass are shown. Each ExPa is depicted on a network diagram by highlighting the reactions active in it. The intensities of green glows around arrows are proportional to the fluxes of the corresponding reactions; numerals alongside the arrows indicate flux values. (A) ExPa A, producing the theoretical maximum DHA yield of $22 \mathrm{~mol} / 100 \mathrm{~mol}$ glucose, without considering cofactor requirement or availability. There is no biomass production and all possible carbon is diverted toward DHA synthesis. (B) ExPa B, producing the theoretical maximum DHA yield of $22 \mathrm{~mol} / 100 \mathrm{~mol}$

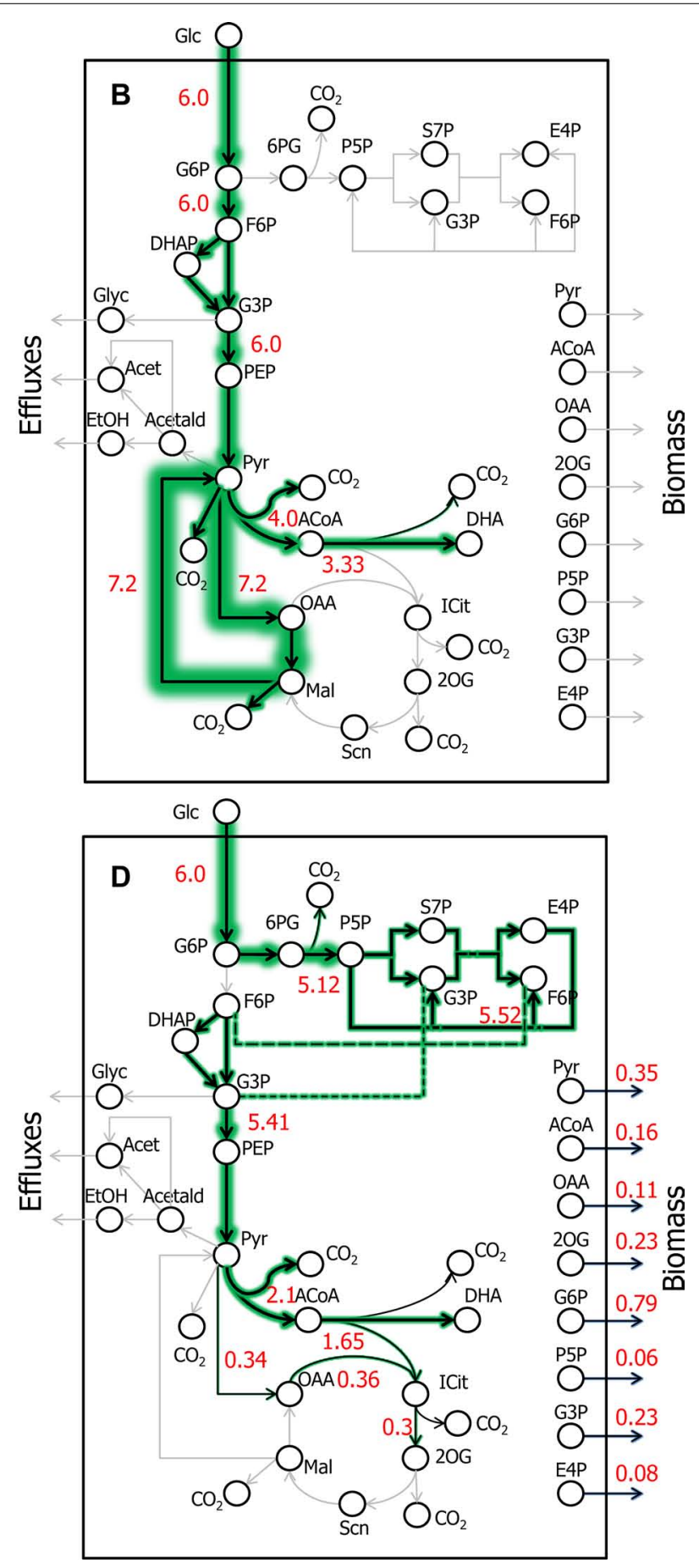

glucose, by accounting for cofactor requirement and availability. There is no biomass production. Here, the NADPH requirement is met by a pyruvate $\gg$ oxaloacetate $\gg$ malate $\gg$ pyruvate shuttle via ME2. (C) ExPa C, producing a high DHA yield of $18 \mathrm{~mol} / 100 \mathrm{~mol}$ glucose, with concomitant biomass production of $3 \mathrm{~mol} / 100 \mathrm{~mol}$ glucose. ME2 supplies reductant to meet the NADPH requirement. (D) ExPa D, producing substantial DHA yield of $11 \mathrm{~mol} / 100 \mathrm{~mol}$ glucose, with concomitant biomass production of $6 \mathrm{~mol} / 100 \mathrm{~mol}$ glucose. Here, the oxidative PPP supplies reductant to meet the NADPH requirement. 
transhydrogenase to interconvert NADH and NADPH. This analysis resulted in 1086 ExPas with 1067 type I, 0 type II, and 19 type III ExPas (Table 1 and Data Sheet 1 in Supplementary Material). Interestingly, the maximal DHA yield obtained from this analysis was identical to the theoretical maximal yield, indicating that some reactions in the network are able to supply the desired cofactors without shunting any carbon away from DHA production. For instance, one $\mathrm{ExPa}(\mathrm{ExPa} \mathrm{B})$ gave a DHA yield of $22 \mathrm{~mol} / 100 \mathrm{~mol}$ glucose by providing NADPH via ME2 with no concomitant biomass production (Figure $3 \mathbf{B}$ ). Here, a pyruvate $\gg$ oxaloacetate $\gg$ malate $\gg$ pyruvate shuttle, with the malate $\gg$ pyruvate conversion catalyzed by ME2, meets the NADPH requirement of DHA synthesis. In all the 238 ExPas that exclusively synthesized DHA, the NADPH requirements were met either by a coupling of the pyruvate carboxylase and ME2 reactions or by operation of the oxidative PPP. In fact, the ME2 reaction for converting malate to pyruvate and the oxidative branch of the PPP are the only two means of producing NADPH in the network. ExPas that recruited the oxidative PPP to meet the NADPH requirement were associated with a DHA yield lower than the theoretical maximum, because the oxidative PPP loses significant carbon as $\mathrm{CO}_{2}$. Consequently, ME2 is preferable to the oxidative PPP as a source of NADPH for DHA synthesis. This makes ME2 an attractive metabolic engineering target.

Consideration of cofactor requirements resulted in an important set of 94 ExPas that simultaneously produced DHA and biomass (Data Sheet 1 in Supplementary Material and Table 1; see examples in Figures 3C,D). These pathways are important to our objective because an overall improvement in DHA yield requires the production of both DHA and biomass at high yields. However, concomitant synthesis of DHA and biomass involves many NADPH-consuming reactions with conflicting NADPH demands. The NADPH demands of DHA biosynthesis are explained earlier in this section; biomass synthesis requires NADPH for the conversion of central carbon metabolic precursors to proteinogenic amino acids and lipogenic fatty acids. Thus, an increase in DHA yield is accompanied by a decrease in biomass yield and vice versa. Two important ExPas that coupled DHA and biomass production are depicted in Figures 3C,D. ExPa C (Figure 3C) produced a high DHA yield of $18 \mathrm{~mol} / 100 \mathrm{~mol}$ glucose, with concomitant biomass production of $3 \mathrm{~mol} / 100 \mathrm{~mol}$ glucose. ME2 supplied reductant to meet the NADPH requirement. ExPa D (Figure 3D) produced substantial DHA yield of $11 \mathrm{~mol} / 100 \mathrm{~mol}$ glucose, with concomitant biomass production of $6 \mathrm{~mol} / 100 \mathrm{~mol}$ glucose. Here, the oxidative PPP supplied reductant to meet the NADPH requirement. Thus, the ExPa analysis provided insights on the capabilities of the DHA biosynthesis metabolic network, and identified ME2 and the oxidative PPP as two critical metabolic engineering targets.

However, ExPa analysis only identifies the modes of the network, as each ExPa is the edge of the realizable flux cone. Because a real flux distribution would be a linear combination of a number of ExPas, it will contain pathways that produce unneeded metabolites. Furthermore, ExPa analysis does not take in to account the carbon requirement for the de novo synthesis of cofactors and energy carriers such as ATP. In the absence of these processes that consume carbon, it overestimates production of biomass and other metabolites compared to experimental results. As a result, the actual improvements in yields would be lower than predicted by the ExPas analysis. To obtain more realistic predictions, we performed flux determination of the DHA-synthesizing strain experimentally using ${ }^{13} \mathrm{C}$ MFA.

\section{${ }^{13} \mathrm{C}$ MFA AND EXTRACELLULAR MEASUREMENTS OF DHA-SYNTHESIZING STRAIN REVEAL NEGLIGIBLE PPP AND LOW TCA CYCLE FLUXES AS WELL AS SUBSTANTIAL FERMENTATIVE FLUXES}

We performed comparative ${ }^{13} \mathrm{C}$ MFA of the three yeast strains $\mathrm{Fy}_{0}, \mathrm{Fy}_{\mathrm{AA}}$, and Fy $\mathrm{DHA}_{\mathrm{H}}$. First, we used RT-PCR to verify that these strains correctly express the recombinant genes engineered into them (Figure 4A). We also verified the strains produce the secondary metabolites expected of them: FyA produced AA but not DHA, FyDHA produced both DHA and AA, whereas Fy F $_{0}$ produced neither compound (data not shown). Figure 4B depicts transient DHA production by Fy these strains (Figure 4C) showed that $\sim 50 \%$ of the consumed galactose was directed toward ethanol, $1 \%$ toward glycerol and less than $10 \%$ toward biomass. Thus, despite aerobic conditions, the strains exhibited substantial fermentative metabolism. Therefore, an initial strategy for improving DHA yields should be the deletion of the ethanol fermentation pathway, which can be expected to double the carbon available for DHA and biomass production.

For a more profound understanding of the metabolic behavior of these strains, we performed ${ }^{13} \mathrm{C}$ MFA on them. Figure 5 depicts key isotopomer trends that emerged from the ${ }^{13} \mathrm{C}$ labeling experiments. Surprisingly, we did not observe any major significant differences in the amino acid isotopomer abundances between the three strains (Data Sheet 2 in Supplementary Material). This was confirmed by a principal component analysis of the isotopomer abundance data, in which all three strains clustered quite close together, although the FyA and Fy $_{\text {DHA }}$ and strains were slightly closer to each other than each was to the control strain $\mathrm{Fy}_{0}$ (data not shown). Figure 6 presents the flux maps obtained from ${ }^{13} \mathrm{C}$ MFA, using the metabolic model listed in Data Sheet 2 in Supplementary Material. This model did not consider cofactors. NADPH and other cofactors are often consumed in processes such as scavenging reductive oxygen species, making it difficult to estimate fluxes in real cellular scenarios. Because ${ }^{13} \mathrm{C}$ MFA obtains significant flux information from isotopomer abundance data, it is a powerful method of estimating fluxes independent of cofactors.

${ }^{13} \mathrm{C}$ MFA revealed that almost all the galactose entering the cell was metabolized via glycolysis with $\sim 10 \%$ of it passing through the oxidative PPP (Figure 6). This is clear from the MIDs of the fragment of alanine in the $100 \% 1-{ }^{13} \mathrm{C}$ galactose labeling experiment (Figures 5A,B and Data Sheet 2 in Supplementary Material). The [123] fragment of pyruvate (and of alanine, which is derived from it) is synthesized from the [321] and [456] fragments of galactose. The oxidative PPP results in the loss of the C-1 carbon of galactose. Thus, alanine synthesized from carbon that passed through the oxidative PPP should exhibit a [123] fragment with the MID $\{m+0: 100 \% ; m+1: 0 \% ; m+2: 0 \% ; m+3: 0 \%\}$. Conversely, alanine synthesized from carbon that passed through glycolysis should exhibit a [123] fragment with the MID $\{m+0: 50 \% ; m+1$ : $50 \% ; m+2: 0 \% ; m+3: 0 \%\}$. In our $100 \% 1-{ }^{13} \mathrm{C}$ galactose labeling experiment, we observed an MID of $\{m+0: 52 \% ; m+1: 46 \%$; 


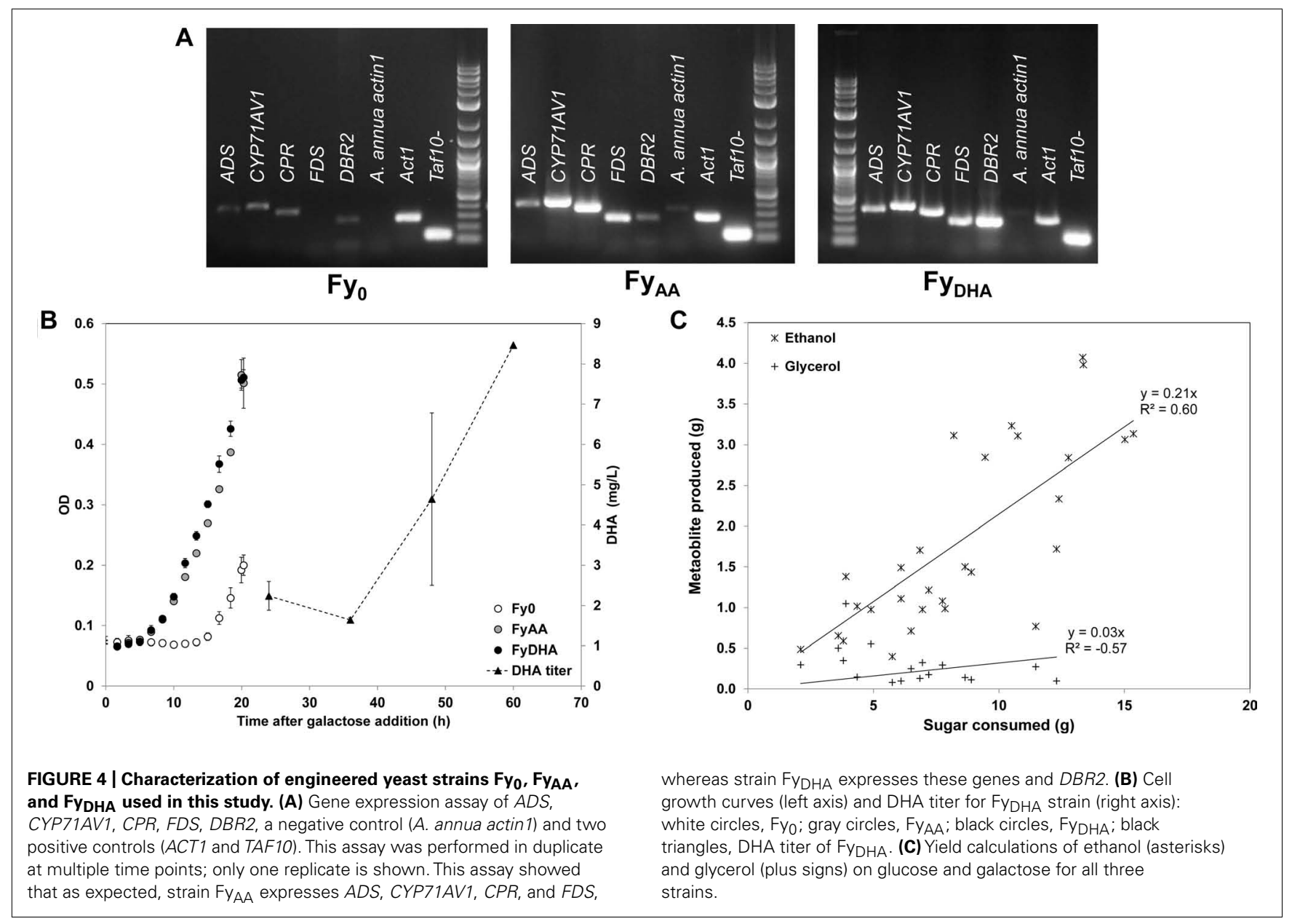

$m+2: 1 \% ; m+3: 0 \%\}$ for the alanine [123] fragment (Figure 5B), indicating that the oxidative PPP was nearly inactive. Data from other amino acid fragments in this experiment as well as the $20 \%$ $\mathrm{U}^{13} \mathrm{C}$ galactose labeling experiment also concur with this result. Because the oxidative PPP generates two NADPH molecules for every molecule of glucose-6-phosphate metabolized through it and is featured in many DHA-synthesizing ExPas identified above, amplifying flux through it is a key metabolic engineering strategy toward improving DHA yield. A possible candidate gene for overexpression is glucose-6-phosphate dehydrogenase $\mathrm{G} 6 \mathrm{PDH} 2$, which is known to be the rate-limiting enzyme in this pathway in S. cerevisiae (Kwon et al., 2006).

Furthermore, the isotopomer data revealed that the TCA cycle carried lower flux than expected under aerobic conditions (Figures 5C,D). This is evident from the MIDs of the aspartic acid fragment [1234] $\{m+0: 66 \% ; m+1: 15 \% ; m+2: 3 \% ; m+3$ : $14 \% ; m+4: 2 \%\}$ and alanine fragment [123] $\{m+0: 78 \% ; m+1$ : $3 \% ; m+2: 1 \% ; m+3: 18 \%$ from the $20 \%$ U- ${ }^{13} \mathrm{C}$ galactose labeling experiment (Figure 5D and Data Sheet 2 in Supplementary Material). Assuming that the ${ }^{13} \mathrm{C}$ enrichment of intracellular $\mathrm{CO}_{2}$ is approximately the same as that of the supplied galactose, synthesis of the fragment [1234] of the TCA cycle intermediate oxaloacetate, and thereby of aspartic acid, purely through the anaplerotic reaction pyruvate $+\mathrm{CO}_{2} \gg$ oxaloacetate should result in the MID $\{m+0: 64 \% ; m+1: 16 \% ; m+2: 0 \% ; m+3: 16 \% ; m+4: 4 \%\}$ for the aspartic acid [1234] fragment. Synthesis of oxaloacetate through the TCA cycle itself should result in a very different MID because of the carbon atom rearrangements in that pathway (Figure 5D). The closeness of the aspartic acid [1234] fragment MID to that predicted through pure anaplerotic synthesis indicates that the TCA cycle has very little flux relative to the anaplerotic reactions or glycolysis (Figure 6).

\section{COMPARISON OF ${ }^{13} \mathrm{C}$ MFA AND EXPa FLUX DISTRIBUTIONS IDENTIFIES FURTHER METABOLIC ENGINEERING TARGETS}

The selection of a preferred DHA-producing ExPa from amongst the 1000+ ExPas elucidated by us would depend on the ease with which the FyDHA strain can be engineered to emulate the ExPa. Therefore, as a step to identify gene targets for improved DHA production, we compared the flux distribution of strain FyDHA and the desired DHA- and biomass-synthesizing ExPas $\mathrm{C}$ and $\mathrm{D}$ from Figures 3C,D (Table 2). Because these ExPas are optimal DHA- and biomass-producing modes, emulating them should result in an improvement of overall DHA yield. Gene overexpressions necessary for FyDHA to emulate ExPa C include pyruvate dehydrogenase, ME2 and pyruvate carboxylase. Genetic interventions necessary to emulate ExPa D include overexpression of pyruvate dehydrogenase, glucose-6-phosphate 


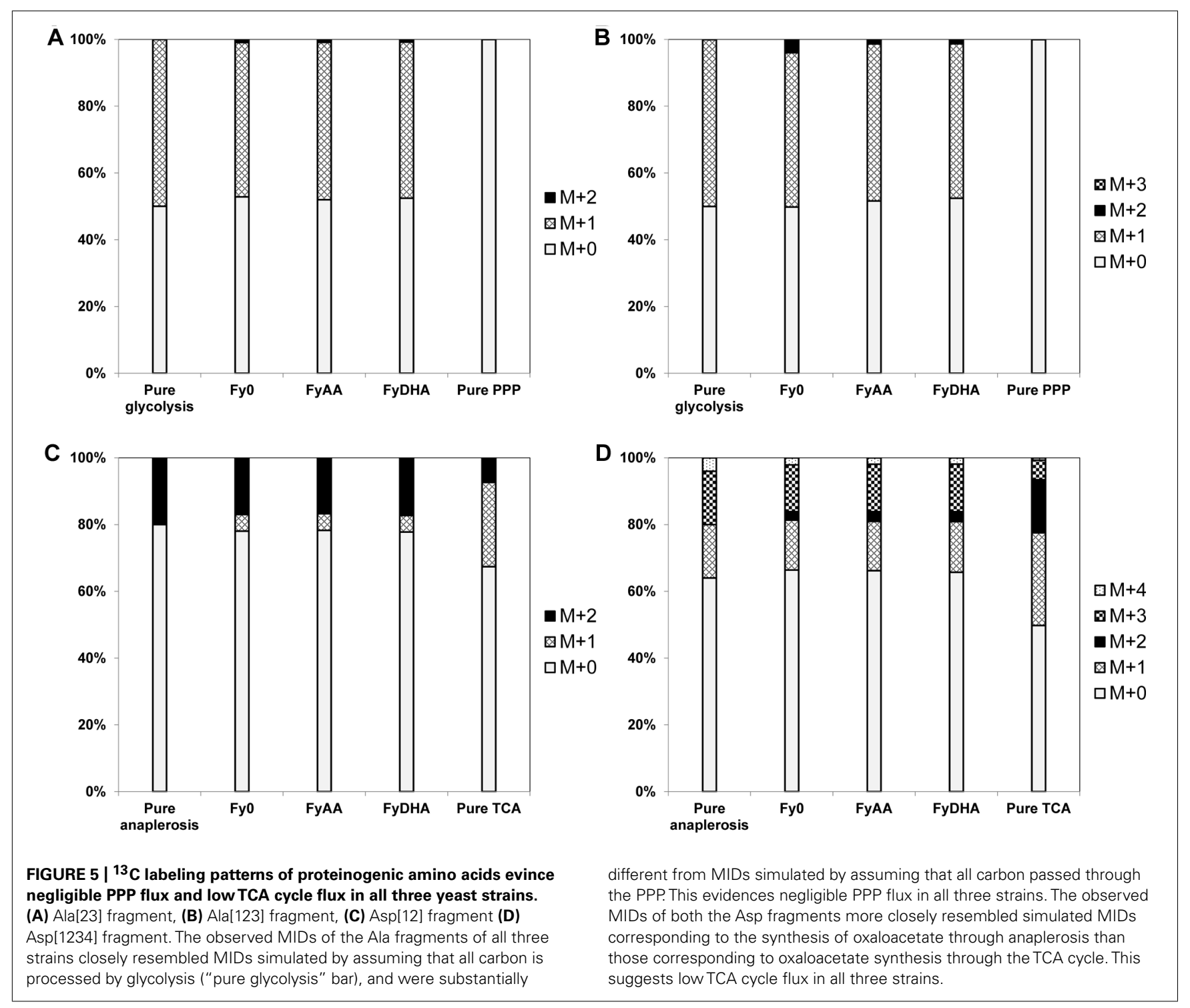

dehydrogenase, transaldolase, and transketolase as well as knockdown of glucose-6-phosphate isomerase (Table 2). Common to both ExPas is pyruvate dehydrogenase, which catalyzes the pyruvate $\gg$ acetyl-CoA reaction. The rationale for overexpressing it is that the DHA-synthesizing strain has a low flux from pyruvate to acetyl-CoA, which would limit the carbon available for DHA production, as acetyl-CoA is the precursor for the MVA pathway. A strong alternative to pyruvate dehydrogenase would be acetyl-CoA synthetase, which plays a role in the pyruvate $\gg$ acetaldehyde $\gg$ acetate $\gg$ acetyl-CoA route that is reported to be a major source of acetyl-CoA in S. cerevisiae (Hynes and Murray, 2010). Another alternative may be ATP citrate lyase, which produces acetyl-CoA from citrate. However, this pathway may not be a major source of cytosolic acetyl-CoA in S. cerevisiae (Hynes and Murray, 2010).

Other gene targets in Table 2 belong to two categories: one involves improving flux through the PPP (overexpression of glucose-6-phosphate dehydrogenase, transketolase, transaldolase)

and reducing the glycolytic flux (knockdown of glucose-6phosphate isomerase), whereas the other involves increasing flux through the malate $\gg$ pyruvate reaction catalyzed by ME2. Both of these are complementary approaches that look to improve NADPH availability for DHA and biomass production. It should be noted that there is a likelihood of some ME2 flux in the yeast strains of this study. In the model we used for ${ }^{13} \mathrm{C} \mathrm{MFA}$, all anaplerotic flux was consolidated into a single bidirectional reaction maef, because our isotopomer measurements could not resolve the different possibilities for this flux: phosphoenolpyruvate carboxykinase, phosphoenolpyruvate carboxylase, and pyruvate carboxylase (phosphoenolpyruvate or pyruvate $+\mathrm{CO}_{2} \gg$ oxaloacetate) as well as $\mathrm{NADH}$-dependent and NADPH-malic enzyme (pyruvate $+\mathrm{CO}_{2} \ll \gg$ malate). The flux distributions returned by ${ }^{13} \mathrm{C}$ MFA included a low net flux (0.02) but substantial reversibility $(0.97-0.98)$ for this consolidated anaplerotic flux, translating to forward $(0.78)$ and reverse $(0.76)$ fluxes that were significant, yet an order of magnitude smaller than 


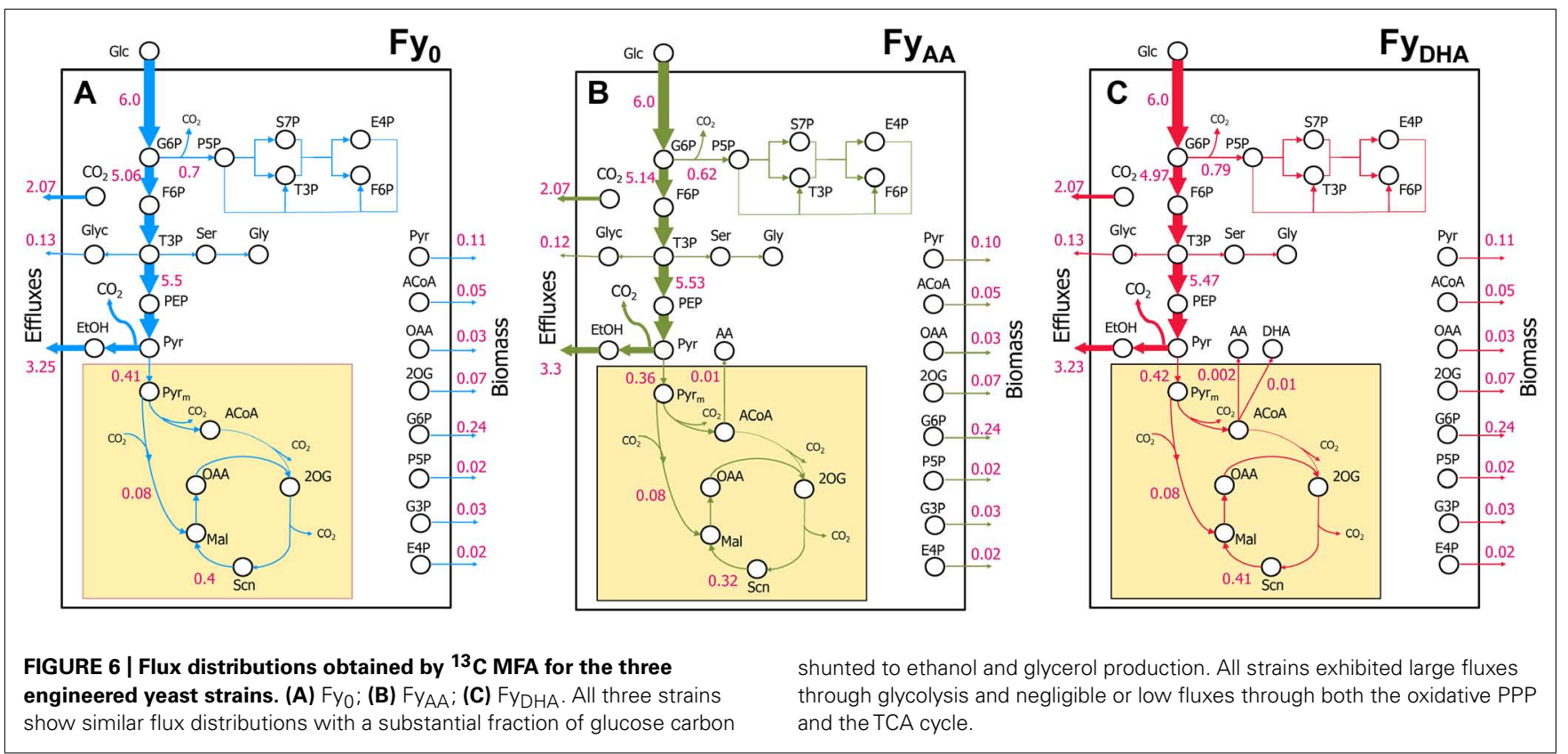

Table 2 | Genetic engineering targets determined by comparing ExPa analysis with ${ }^{13} \mathrm{C}$ MFA.

\begin{tabular}{lll}
\hline & ExPa C & ExPa D \\
\hline DHA yield & 0.18 & 0.11 \\
Biomass yield & 0.03 & 0.06 \\
NADPH supplied by & ME2 & PPP \\
Gene overexpression in strain & & \\
FyDHA necessary to emulate & 1. YBR221C, YER178W, YFL018C, YGR193C, YNLO71W & 1. YBR221C, YER178W, YFLO18C, YGR193C, YNLO71W \\
this ExPa & (pyruvate dehydrogenase) & (pyruvate dehydrogenase) \\
& 2. YKL029C (NADPH-malic enzyme ME2) & 2. YNL241C (glucose-6-phosphate dehydrogenase) \\
& 3. YBR218C, YGL062W (pyruvate carboxylase) & 3. YGR043C, YLR354C (transaldolase in PPP) \\
& & 4. YBR117C, YPR074C (transketolase in PPP) \\
Gene knockdown in strain FyDHA & 1. YBR145W, YGL256W, YOL086C (alcohol & 1. YBR145W, YGL256W, YOLO86C(alcohol dehydrogenase) \\
necessary to emulate this ExPa & dehydrogenase) & 2. YBR196C (glucose-6-phosphate isomerase)
\end{tabular}

The characteristics of two key ExPas with reasonable DHA and biomass yields are shown, with the corresponding genetic engineering targets to emulate these ExPas in Fy DHA. Common targets include eliminating ethanol biosynthesis by deleting alcohol dehydrogenase and increasing flux from pyruvate to acetyl-CoA by overexpressing pyruvate dehydrogenase.

the glycolytic flux $(\sim 5.0$; Data Sheet 2 in Supplementary Material and Figure 6). ME2 may contribute to this flux, although this contribution remains unresolved. However, a comparison with $\mathrm{ExPa} \mathrm{B}$ or C (Figures 3B,C) shows that even a $100 \%$ contribution by ME2 to this flux is insufficient to meet NADPH demand.

\section{FBA AND MOMA ANALYSIS OF THE MODIFIED IMM904 GENOME-SCALE MODEL REVEAL MULTIPLE GENETIC INTERVENTION STRATEGIES FOR IMPROVING DHA YIELDS}

Flux balance analysis, a complementary approach to ${ }^{13} \mathrm{CMFA}$, estimates or predicts fluxes using linear programming techniques. It is particularly useful for analysis of metabolism at the genome-scale. MOMA is an extension of the FBA approach that identifies nontrivial gene knockouts toward optimizing a metabolic objective, such as the yield of a product. Its use toward determining gene targets has been reported in multiple successful studies (Alper et al., 2005; Park et al., 2007; Asadollahi et al., 2009; Ranganathan et al., 2012). We performed genome-scale FBA and MOMA analysis by using a modified iMM904 model (Mo et al., 2009). First, we used the fluxes obtained from ${ }^{13} \mathrm{C}$ MFA of the Fy $\mathrm{y}_{\mathrm{DHA}}$ strain for constraining fluxes in the iMM904 model, and then used FBA to predict a flux distribution for this strain. Then, we used MOMA analysis to simulate fluxes in single- and double-gene knockouts 
of the FyDHA strain to study the effects of these knockouts on DHA yields.

We initially observed that even with double-gene knockouts, DHA yields did not improve significantly over those of FyDHA. This is because MOMA minimizes flux changes between the knocked out and control strains, and the low fluxes of NADPHsupplying reactions in the FyDHA strain prevented MOMA from identifying knockouts for improving DHA yield. Consequently, we modified the flux distribution of FyDHA in silico such that it had equal fluxes through PPP and glycolysis, but its DHA yield was the same as the measured yield of FyDHA. We performed subsequent MOMA analysis on this new baseline strain FyDHA' Subsequent MOMA analysis found 401 single-gene knockouts with significantly increased DHA flux compared to FyDHA' We then used the single-gene knockouts associated with the highest DHA yields to identify double-gene knockouts that would potentially exhibit even higher DHA yields. The MOMA analysis predicted substantially improved DHA yields in these knockouts (Data Sheet 3 in Supplementary Material). In many cases, the increase in DHA yield was accompanied by an expected decrease in biomass yield, because knocking out genes can lead to suboptimal growth as the products of the knocked out genes are no longer available for biomass synthesis. A good gene knockout strategy should improve DHA yields substantially with a modest decrease in biomass growth rate.

The best double-gene knockout strategy predicted by MOMA includes the genes YKL120W and YKL106W, and improves DHA yield to $4.2 \mathrm{~mol} \%$ (70-fold over the baseline), while reducing biomass yield by only 2.2-fold compared to FyDHA' Figure 7 presents an interpretation of this strategy. First, FBA predicted

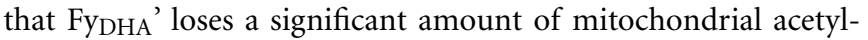
CoA to $\mathrm{CO}_{2}$ through a cyclic, cytosolic pathway that contains most steps of the TCA cycle. Both YKL120W and YKL106W code for enzymes or transporter proteins in facilitating this cycle. YKL120W or $O A C 1$ codes for an oxaloacetate transporter catalyzing the transport oxaloacetate $[\mathrm{c}]+\mathrm{H}^{+}[\mathrm{c}] \gg$ oxaloacetate $[\mathrm{m}]+\mathrm{H}^{+}[\mathrm{m}]$, where $[\mathrm{c}]$ and $[\mathrm{m}]$ denote the cytosol and mitochondrion, respectively. YKL106W or AAT1 codes for an aspartate aminotransferase catalyzing the reaction $\alpha$-ketoglutarate $[\mathrm{m}]+\mathrm{L}$-aspartate $[\mathrm{m}] \gg \mathrm{L}$ glutamate $[\mathrm{m}]+$ oxaloacetate $[\mathrm{m}]$. Thus, knocking out YKL120W and YKL106W breaks this cycle while not affecting biomass production, allowing mitochondrial acetyl-CoA to be diverted toward downstream pathways including DHA biosynthesis. Of these two genes, YKL120W has a greater effect on reducing flux toward

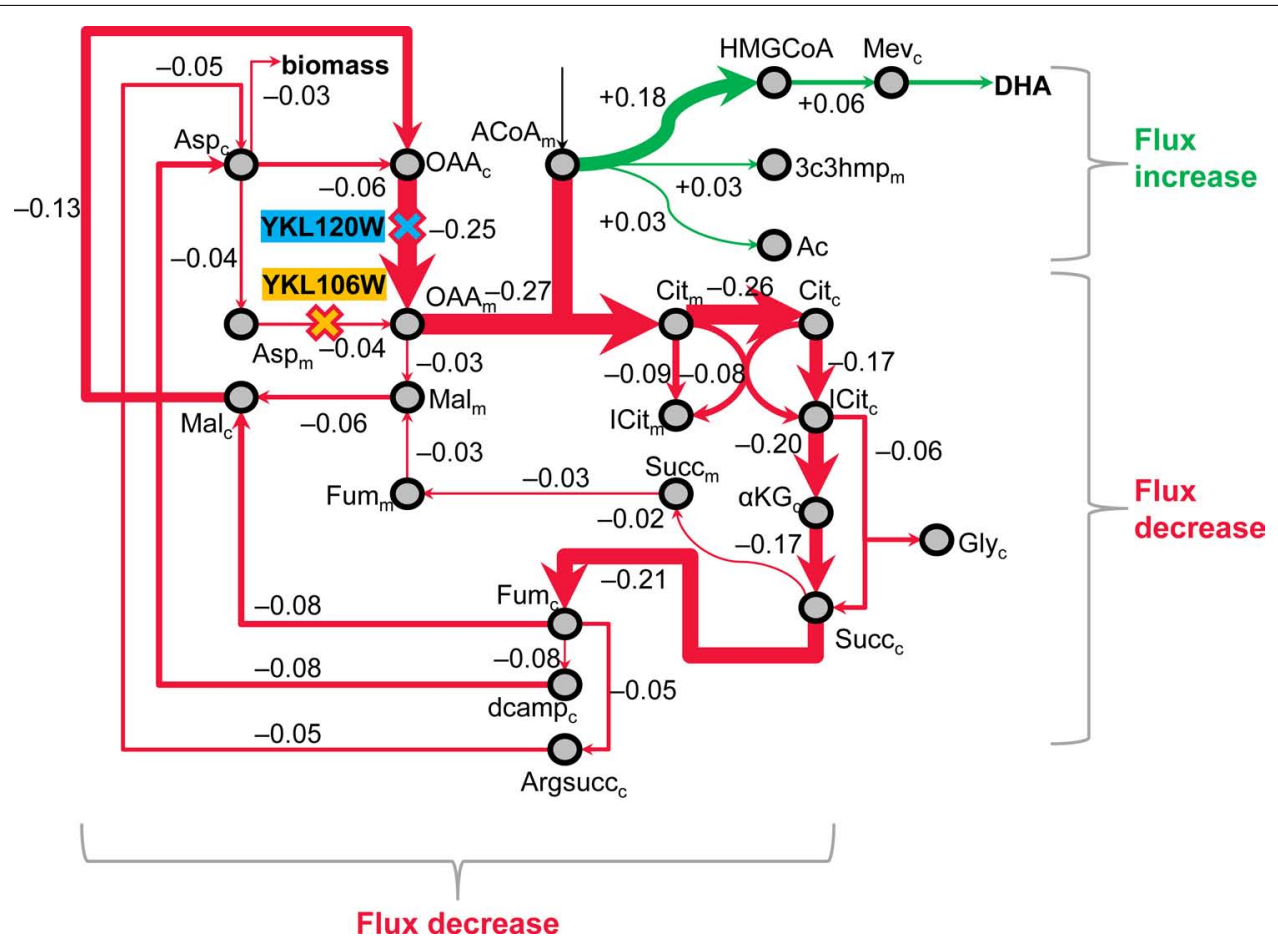

FIGURE 7 | MOMA analysis identifies double-gene knockouts in primary metabolism that funnel carbon toward DHA. As per one MOMA prediction, engineering the Fy $y_{D H A}$ strain by knocking out two genes (1: OAC1 or YKL120W, which codes for an oxaloacetate transporter and 2: AAT1 or YKL106W, which codes for an aspartate aminotransferase) should result in $4.2 \mathrm{~mol}$ DHA per $100 \mathrm{~mol}$ of glucose, which amounts to a $\sim 70$-fold increase as compared to our current yield and translates to $\sim 18 \%$ of the theoretical yield of DHA from glucose. This metabolic map depicts the mechanism by which these two deletions accomplish the flux redirection. Red arrows indicate decreased flux and green arrows indicate increase in flux relative to our (control) DHA-producing strain; arrow thicknesses and numbers alongside the arrows represent flux differences between the baseline strain Fy ${ }_{D H A}$ ' and the double-gene knockout strain are in arbitrary units. On the basis of the ${ }^{13} \mathrm{C}$ isotopomer results, FBA predicted that our control strain loses a significant amount of mitochondrial acetyl-CoA ( $\mathrm{ACo} \mathrm{Am}_{\mathrm{m}}$ ) to $\mathrm{CO}_{2}$ through a cyclic, cytosolic pathway that contains most steps of the TCA cycle. Knocking out YKL120W and YKL106W breaks this cycle while not significantly affecting biomass production, thus allowing $A C o A_{m}$ to be diverted toward downstream pathways including DHA biosynthesis. 
mitochondrial oxaloacetate, and also shows up as part of the next best double-gene knockout pair for improving DHA yield (Data Sheet 3 in Supplementary Material).

A limitation of using FBA-type methodologies to predict genetic engineering strategies is their reliance of the assumption of optimized metabolism to predict flux distributions. However, engineered strains may perform sub-optimally. Thus, while FBA is valuable in identifying non-trivial gene targets, it is ambitious to expect the real engineered strain to exhibit yields close to the FBA-predicted yields. Additionally, a recent statistical analysis of previous studies on the production of chemicals by engineered $S$. cerevisiae found that genetic intervention improved product yield only by two- to fourfold in a majority of the 40 studies examined, and that chemicals requiring multiple enzymatic steps were associated with lower yields (Varman et al., 2011). Along the same lines, implementation of the best gene knockout strategy suggested by our study may result in a DHA yield improvement tangibly lower than the predicted 70 -fold increase. Very likely, one round of genetic engineering in accordance with FBA predictions may necessitate further rounds of engineering to overcome further bottlenecks to product yield.

\section{CONCLUSION}

In summary, we investigated various in silico and in vivo approaches - ExPa analysis, ${ }^{13} \mathrm{C}$ MFA, FBA, and MOMA analysis - to identify metabolic engineering strategies for improving DHA yield in an engineered yeast strain. Our analyses revealed three major metabolic engineering strategies toward this objective. The first, most obvious strategy is reduction of flux from pyruvate to ethanol and directing this flux toward acetyl-CoA by knocking out fermentative pathways and overexpressing pyruvate dehydrogenase. However, in the absence of further genetic intervention, the available carbon is not likely to be redirected toward the desired pathway. A thorough investigation of the system using detailed ${ }^{13} \mathrm{C}$ MFA, and FBA and MOMA analysis was required to decipher further non-intuitive strategies. A second strategy toward higher DHA yield is the improvement of NADPH availability by overexpressing $M E 2$ or a rate-limiting step of the

\section{REFERENCES}

Ahn, W. S., and Antoniewicz, M. R. (2013). Parallel labeling experiments with [1,2-13C]glucose and [U13C]glutamine provide new insights into $\mathrm{CHO}$ cell metabolism. Metab. Eng. 15, 34-47. doi: 10.1016/ j.ymben.2012.10.001

Alper, H., Jin, Y.-S., Moxley, J. F., and Stephanopoulos, G. (2005). Identifying gene targets for the metabolic engineering of lycopene biosynthesis in Escherichia coli. Metab. Eng. 7, 155-164. doi: 10.1016/j.ymben.2004.12.003

Asadollahi, M. A., Maury, J., Patil, K. R., Schalk, M., Clark, A., and Nielsen, J. (2009). Enhancing sesquiterpene production in Saccharomyces cerevisiae through in silico driven metabolic engineering. Metab.
Eng. 11, 328-334. doi: 10.1016/ j.ymben.2009.07.001

Ba, Q., Zhou, N., Duan, J., Chen, T. Hao, M., Yang, X., et al. (2012). Dihydroartemisinin exerts its anticancer activity through depleting cellular iron via transferrin receptor- 1 . PLoS ONE 7:e42703. doi: 10.1371/journal.pone.0042703

Becker, J., Zelder, O., Häfner, S., Schröder, H., and Wittmann, C. (2011). From zero to hero design-based systems metabolic engineering of Corynebacterium glutamicum for L-lysine production. Metab. Eng. 13, 159-168. doi: 10.1016/j.ymben.2011.01.003

Bell, S. L., and Palsson, B. Ø. (2004) Expa: a program for calculating extreme pathways in biochemical reaction networks. Bioinformatics 21, oxidative PPP such as G6PDH2. Harnessing the oxidative PPP for NADPH supply suffers from the disadvantage that a sixth of the carbon entering this pathway is lost as $\mathrm{CO}_{2}$; hence, this lowers the yield of DHA significantly. A third strategy is pushing flux from mitochondrial acetyl-CoA toward DHA production. These two strategies are necessary for overcoming the NADPH and mitochondrial acetyl-CoA availability bottlenecks en route to DHA biosynthesis. We anticipate that comprehensive analysis of a similar type can be applied to any metabolic system to reveal intuitive and non-intuitive genetic engineering targets for optimizing the production of a target molecule. Such systemic analysis is an important step toward realizing the goal of rational metabolic engineering.

\section{CONTRIBUTIONS}

Ashish Misra, Matthew F. Conway, Eddy C. Agbo, and Ganesh Sriram conceived this work; Ashish Misra, Matthew F. Conway, Joseph Johnnie, and Ganesh Sriram designed it. Ashish Misra, Matthew F. Conway, and Joseph Johnnie performed computational analyses; Matthew F. Conway and Ashish Misra performed ${ }^{13} \mathrm{C}$ labeling experiments and ${ }^{13} \mathrm{C}$ MFA. Tabish M. Qureshi performed RT-PCR and DHA assays. Ashish Misra and Ganesh Sriram interpreted the results, prepared data displays, wrote the manuscript and revised the manuscript.

\section{ACKNOWLEDGMENTS}

The authors would like to thank Elena Chung and Eli Tung, Department of Chemical and Biomolecular Engineering, University of Maryland, for assistance with experimental analyses. This work was funded by Maryland Industrial Partnerships (MIPS; award no. 4426). Matthew F. Conway was partially supported by a Howard Hughes Medical Institute Undergraduate Research Fellowship from University of Maryland.

\section{SUPPLEMENTARY MATERIAL}

The Supplementary Material for this article can be found online at http://www.frontiersin.org/Microbiotechnology,_Ecotoxicology_ and_Bioremediation/10.3389/fmicb.2013.00200/abstract
1739 -1740. doi: 10.1093/bioinformatics/bti228

Bertea, C. M., Freije, J. R., van der Woude, H., Verstappen, F. W., Perk, L., Marquez, V., et al. (2005). Identification of intermediates and enzymes involved in the early steps of artemisinin biosynthesis in Artemisia annua. Planta Med. 71, 40-47. doi: 10.1055/s-2005-837749

Brown, G. D., and Sy, L.-K. (2004). In vivo transformations of dihydroartemisinic acid in Artemisia annua plants. Tetrahedron 60, 11391159. doi: 10.1016/j.tet.2003.11.070 Bruinenberg, P. M., Dijken, J. P. V., and Scheffers, W. A. (1983). A theoretical analysis of NADPH production and consumption in yeasts. $J$. Gen. Microbiol. 129, 953-964. doi: 10.1099/00221287-129-4-953
Burgard, A. P., Pharkya, P., and Maranas, C. D. (2003). Optknock: a bilevel programming framework for identifying gene knockout strategies for microbial strain optimization. Biotechnol. Bioeng. 84, 647-657. doi: 10.1002/bit.10803

Chandran, D., Copeland, W. B., Sleight, S. C., and Sauro, H. M. (2008). Mathematical modeling and synthetic biology. Drug Discov. Today Dis. Models 5, 299-309. doi: 10.1016/j.ddmod.2009.07.002

Covello, P. S. (2008). Making artemisinin. Phytochemistry 69, 28812885. doi: 10.1016/j.phytochem. 2008.10.001

Eastman, R. T., and Fidock, D. A. (2009). Artemisinin-based combination therapies: a vital tool in efforts to eliminate malaria. Nat. 
Rev. Microbiol. 7, 864-874. doi: 10.1038/nrmiro2239

Feachem, R. G., Phillips, A. A., Hwang, J., Cotter, C., Wielgosz, B., Greenwood, B. M., et al. (2010). Shrinking the malaria map: progress and prospects. Lancet 376 , 1566-1578. doi: 10.1016/S0140-6736 (10)61270-6

Feng, X., Tang, K.-H., Blankenship, R. E., and Tang, Y. J. (2010). Metabolic flux analysis of the mixotrophic metabolisms in the green sulfur bacterium Chlorobaculum tepidum. J. Biol. Chem. 285, 39544-39550. doi: 10.1074/jbc.M110.162958

Ferreira, J. F. S., Luthria, D. L., Sasaki, T., and Heyerick, A. (2010). Flavonoids from Artemisia annua L. as antioxidants and their potential synergism with artemisinin against malaria and cancer. Molecules 15, 3135-3170. doi: 10.3390/molecules15053135

Gao, W., Xiao, F., Wang, X., and Chen, T. (2013). Artemisinin induces A549 cell apoptosis dominantly via a reactive oxygen species-mediated amplification activation loop among caspase-9, -8 and -3. Apoptosis doi: 10.1007/s10495-013-0857-z [Epub ahead of print].

Grafahrend-Belau, E., Schreiber, F., Koschutzki, D., and Junker, B. H. (2009). Flux balance analysis of barley seeds: a computational approach to study systemic properties of central metabolism. Plant Physiol. 149, 585598. doi: 10.1104/pp.108.129635

Hale, V., Keasling, J., Renninger, N., and Diagana, T. (2007). "Microbially derived artemisinin: a biotechnology solution to the global problem of access to affordable antimalarial drugs," in Defining and Defeating the Intolerable Burden of Malaria III: Progress and Perspectives, eds J. Breman, M. Alilio, and N. White (Northbrook: American Society of Tropical Medicine and Hygiene).

Hynes, M. J., and Murray, S. L. (2010). ATP-citrate lyase is required for production of cytosolic acetyl coenzyme A and development in Aspergillus nidulans. Eukaryot. Cell 9, 10391048. doi: 10.1128/EC.00080-10

Kindermans, J.-M., Pilloy, J., Olliaro, P., and Gomes, M. (2007). Ensuring sustained ACT production and reliable artemisinin supply. Malar. J. 6, 125. doi: 10.1186/1475-28756-125

Kwon, D.-H., Kim, M.-D., Lee, T.H., Oh, Y.-J., Ryu, Y.-W., and Seo, J.-H. (2006). Elevation of glucose 6-phosphate dehydrogenase activity increases xylitol production in recombinant Saccharomyces cerevisiae. J. Mol. Catal. B Enzym. 43,
86-89. doi: 10.1016/j.molcatb.2006. 06.014

Lenihan, J. R., Tsuruta, H., Diola, D. Renninger, N. S., and Regentin, R. (2008). Developing an industrial artemisinic acid fermentation process to support the costeffective production of antimalarial artemisinin-based combination therapies. Biotechnol. Prog. 24, 10261032. doi: 10.1002/btpr.27

Lévesque, F., and Seeberger, P. H (2012). Continuous-flow synthesis of the anti-malaria drug artemisinin. Angew. Chem. Int. Ed. Engl. 51, 17061709. doi: 10.1002/anie.201107446

Liu, N. Q., Schuehly, W., von Freyhold, M., and van der Kooy, F. (2011). A novel purification method of artemisinin from Artemisia annua. Ind. Crops Prod. 34, 1084-1088. doi: 10.1016/j.indcrop.2011.03.023

Llaneras, F., and Picó, J. (2010). Which metabolic pathways generate and characterize the flux space? A comparison among elementary modes, extreme pathways and minimal generators. J. Biomed. Biotechnol. 2010, 753904 doi: 10.1155/2010/753904

Mo, M. L., Palsson, B. O., and Herrgård, M. J. (2009). Connecting extracellular metabolomic measurements to intracellular flux states in yeast. $B M C$ Syst. Biol. 3:37. doi: 10.1186/17520509-3-37

Murphy, T. A., Dang, C. V., and Young, J. D. (2013). Isotopically nonstationary $13 \mathrm{C}$ flux analysis of Mycinduced metabolic reprogramming in B-cells. Metab. Eng. 15, 206-217. doi: 10.1016/j.ymben.2012.07.008

Nargund, S., and Sriram, G. (2013). Designer labels for plant metabolism: statistical design of isotope labeling experiments for improved quantification of flux in complex plant metabolic networks. Mol. Biosyst. 9, 99-112. doi: 10.1039/ c2 $\mathrm{mb} 25253 \mathrm{~h}$

Nissen, T. L., Anderlund, M., Nielsen, J., Villadsen, J., and KiellandBrandt, M. C. (2001). Expression of a cytoplasmic transhydrogenase in Saccharomyces cerevisiae results in formation of 2-oxoglutarate due to depletion of the NADPH pool. Yeast 18, 19-32. doi: 10.1002/10970061(200101)18:1

O’Meara, W. P., Mangeni, J. N., Steketee, R., and Greenwood, B. (2010). Changes in the burden of malaria in sub-Saharan Africa. Lancet Infect. Dis. 10, 545-555. doi: 10.1016/S1473-3099(10)70096-7

Orth, J. D., Thiele, I., and Palsson, B. O. (2010). What is flux balance analysis? Nat. Biotechnol. 28, 245-248. doi: $10.1038 /$ nbt. 1614
Paddon, C. J., Westfall, P. J., Pitera, D. J., Benjamin, K., Fisher, K., McPhee, D., et al. (2013). High-level semisynthetic production of the potent antimalarial artemisinin. Nature advance online publication. Available at: http://www.nature.com/nature/ journal/vaop/ncurrent/full/nature12 051.html [accessed April 14, 2013].

Palsson, B. O. (2006). Systems Biology: Properties of Reconstructed Networks. New York: Cambridge University Press.

Papini, M., Nookaew, I., Uhlén, M., and Nielsen, J. (2012). Scheffersomyces stipitis: a comparative systems biology study with the Crabtree positive yeast Saccharomyces cerevisiae. Microb. Cell Fact. 11, 136. doi: 10.1186/1475-2859-11-136

Park, J. H., Lee, K. H., Kim, T. Y., and Lee, S. Y. (2007). Metabolic engineering of Escherichia coli for the production of l-valine based on transcriptome analysis and in silico gene knockout simulation. Proc. Natl. Acad. Sci. U.S.A. 104, 7797-7802. doi: 10.1073/pnas.07026 09104

Pharkya, P., Burgard, A. P., and Maranas, C. D. (2004). OptStrain: a computational framework for redesign of microbial production systems. Genome Res. 14, 2367-2376. doi: 10.1101/gr.2872004

Price, R. N., and Douglas, N. M. (2009). Artemisinin combination therapy for malaria: beyond good efficacy. Clin. Infect. Dis. 49, 1638-1640. doi: 10.1086/647947

Ranganathan, S., Suthers, P. F., and Maranas, C. D. (2010). Optforce: an optimization procedure for identifying all genetic manipulations leading to targeted overproductions. PLoS Comput. Biol. 6 : e1000744. doi: 10.1371/journal.pcbi. 1000744

Ranganathan, S., Tee, T. W., Chowdhury, A., Zomorrodi, A. R., Yoon J. M., Fu, Y., et al. (2012). An integrated computational and experimental study for overproducing fatty acids in Escherichia coli. Metab. Eng. 14, 687-704. doi: 10.1016/ j.ymben.2012.08.008

Ro, D.-K., Paradise, E. M., Ouellet, M., Fisher, K. J., Newman, K. L., Ndungu, J. M., et al. (2006). Production of the antimalarial drug precursor artemisinic acid in engineered yeast. Nature 440, 940-943. doi: 10.1038 /nature 04640

Romano, A. H., and Conway, T. (1996). Evolution of carbohydrate metabolic pathways. Res. Microbiol. 147, 448-455. doi: 10.1016/09232508(96)83998-2
Rydén, A.-M., Ruyter-Spira, C., Quax, W. J., Osada, H., Muranaka, T., Kayser, O., et al. (2010). The molecular cloning of dihydroartemisinic aldehyde reductase and its implication in artemisinin biosynthesis in Artemisia annua. Planta Med. 76, 1778-1783. doi: 10.1055/s-00301249930

Schellenberger, J., Que, R., Fleming, R. M. T., Thiele, I., Orth, J. D., Feist, A. M., et al. (2011). Quantitative prediction of cellular metabolism with constraint-based models: the COBRA Toolbox v2.0. Nat. Protoc. 6, 1290-1307. doi: 10.1038/nprot.2011.308

Schilling, C. H., Letscher, D., and Palsson, B. Ø. (2000). Theory for the systemic definition of metabolic pathways and their use in interpreting metabolic function from a pathwayoriented perspective. J. Theor. Biol. 203, 229-248. doi: 10.1006/ jtbi.2000.1073

Schilling, C. H., and Palsson, B. O. (2000). Assessment of the metabolic capabilities of Haemophilus influen$z a e$ Rd through a genome-scale pathway analysis. J. Theor. Biol. 203, 249-283. doi: 10.1006/jtbi.2000.1088 Segrè, D., Vitkup, D., and Church, G. M. (2002). Analysis of optimality in natural and perturbed metabolic networks. Proc. Natl. Acad. Sci. U.S.A. 99, 15112-15117. doi: 10.1073/pnas.232349399

Sriram, G., Fulton, D. B., Iyer, V. V., Peterson, J. M., Zhou, R., Westgate, M. E., etal. (2004). Quantification of compartmented metabolic fluxes in developing soybean embryos by employing biosynthetically directed fractional 13C labeling, two-dimensional [13C, 1H] nuclear magnetic resonance, and comprehensive isotopomer balancing. Plant Physiol. 136, 3043-3057. doi: 10.1104/pp.104.050625

Sriram, G., Rahib, L., He, J.-S., Campos, A. E., Parr, L. S., Liao, J. C., et al. (2008). Global metabolic effects of glycerol kinase overexpression in rat hepatoma cells. Mol. Genet. Metab. 93, 145-159. doi: 10.1016/j.ymgme.2007.09.008

Stephanopoulos, G. N., Aristidou, A. A., and Nielsen, J. (1998). Metabolic Engineering: Principles and Methodologies, 1st Edn, Waltham: Academic Press.

Sy, L.-K., and Brown, G. D. (2002). The mechanism of the spontaneous autoxidation of dihydroartemisinic acid. Tetrahedron 58, 897-908. doi: 10.1016/S0040-4020(01)01193-0

Szyperski, T. (1995). Biosynthetically directed fractional 13C-labeling 
of proteinogenic amino acids. An efficient analytical tool to investigate intermediary metabolism. Eur. J. Biochem. 232, 433-448. doi: 10.1111/j.1432-1033.1995.tb20829.x

Szyperski, T. (1998). ${ }^{13}$ C-NMR, MS and metabolic flux balancing in biotechnology research. Q. Rev. Biophy. 31, 41-106.

Teoh, K., Polichuk, D., Reed, D., Nowak, G., and Covello, P. (2006). Artemisia annua L. (Asteraceae) trichomespecific cDNAs reveal CYP71AV1, a cytochrome $\mathrm{P} 450$ with a key role in the biosynthesis of the antimalarial sesquiterpene lactone artemisinin. FEBS Lett. 580, 1411-1416. doi: 10.1016/j.febslet.2006.01.065

Teste, M.-A., Duquenne, M., François, J. M., and Parrou, J.-L. (2009). Validation of reference genes for quantitative expression analysis by real-time RT-PCR in Saccharomyces cerevisiae. BMC Mol. Biol. 10:99. doi: 10.1186/1471-2199-10-99

Varman, A. M., Xiao, Y., Leonard, E., and Tang, Y. J. (2011). Statisticsbased model for prediction of chemical biosynthesis yield from Saccharomyces cerevisiae. Microb. Cell Fact. 10, 45. doi: 10.1186/1475-2859-10-45 Wallaart, T. E., Pras, N., Beekman, A. C., and Quax, W. J. (2000). Seasonal variation of artemisinin and its biosynthetic precursors in plants of Artemisia annua of different geographical origin: proof for the existence of chemotypes. Planta Med. 66, 57-62. doi: 10.1055/s-2000-11115

Weathers, P., Elkholy, S., and Wobbe, K. (2006). Artemisinin: the biosynthetic pathway and its regulation in Artemisia annua, a terpenoid-rich species. In Vitro Cell. Dev. Biol. Plant 42, 309-317. doi: 10.1079/IVP2006782

Westfall, P. J., Pitera, D. J., Lenihan, J. R., Eng, D., Woolard, F. X., Regentin, R., et al. (2012). Production of amorphadiene in yeast, and its conversion to dihydroartemisinic acid, precursor to the antimalarial agent artemisinin. Proc. Natl. Acad. Sci. U.S.A. 109, E111-E118. doi: 10.1073/pnas.1110740109

Wiback, S. J., and Palsson, B. O. (2002). Extreme pathway analysis of human red blood cell metabolism. Biophys. J. 83, 808818. doi: 10.1016/S0006-3495(02) 75210-7

Wiechert, W. (2001). 13C metabolic flux analysis. Metab. Eng. 3, 195-206. doi: 10.1006/mben.2001.0187

World Health Organization (2010). World Malaria Report 2010. WHO. Available at: http://www.who.int/ malaria/world_malaria_report_2010/ en/index.html [accessed April 14, 2013].

Zhang, Y., Nowak, G., Reed, D. W., and Covello, P. S. (2011). The production of artemisinin precursors in tobacco. Plant Biotechnol. J. 9, 445-454. doi: 10.1111/j.1467-7652.2010.00556.x

Zhang, Y., Teoh, K. H., Reed, D. W., Maes, L., Goossens, A., Olson, D. J. H., etal. (2008). The molecular cloning of artemisinic aldehyde $\Delta 11(13)$ reductase and its role in glandular trichome-dependent biosynthesis of artemisinin in Artemisia annua. J. Biol. Chem. 283, 21501-21508. doi: 10.1074/jbc. M803090200

Zhang, Y. J., Gallis, B., Taya, M., Wang, S., Ho, R. J. Y., and Sasaki, T. (2013). pH-responsive artemisinin derivatives and lipid nanoparticle formulations inhibit growth of breast cancer cells in vitro and induce downregulation of HER family members. PLOS ONE 8:e59086. doi: 10.1371/journal.pone.0059086

Zheng, Y., and Sriram, G. (2010). Mathematical modeling: bridging the gap between concept and realization in synthetic biology. J. Biomed. Biotechnol. 2010, 541609. doi: 10.1155/2010/541609

Zhu, C., and Cook, S. P. (2012). A concise synthesis of $(+)$-artemisinin. J.
Am. Chem. Soc. 134, 13577-13579. doi: $10.1021 /$ ja3061479

Conflict of Interest Statement: Eddy C. Agbo, Anne M. Derrick and Bao Lige are employed with Fyodor Biotechnologies Corp., which has financial interest in the commercialization of antimalarial therapeutics or their precursors. The other authors have no conflict of interest.

Received: 25 April 2013; accepted: 25 June 2013; published online: 26 July 2013.

Citation: Misra A, Conway MF, Johnnie J, Qureshi TM, Derrick AM, Agbo EC and Sriram $G$ (2013) Metabolic analyses elucidate non-trivial gene targets for amplifying dihydroartemisinic acid production in yeast. Front. Microbiol. 4:200. doi: 10.3389/fmicb.2013.00200

This article was submitted to Frontiers in Microbiotechnology, Ecotoxicology and Bioremediation, a specialty of Frontiers in Microbiology.

Copyright () 2013 Misra, Conway, Johnnie, Qureshi, Derrick, Agbo and Sriram. This is an open-access article distributed under the terms of the Creative Commons Attribution License, which permits use, distribution and reproduction in other forums, provided the original authors and source are credited and subject to any copyright notices concerning any thirdparty graphics etc. 